\title{
What the Brain Stem Tells the Frontal Cortex. I. Oculomotor Signals Sent From Superior Colliculus to Frontal Eye Field Via Mediodorsal Thalamus
}

\author{
Marc A. Sommer and Robert H. Wurtz \\ Laboratory of Sensorimotor Research, National Eye Institute, National Institutes of Health, Bethesda, Maryland 20892-4435
}

Submitted 31 July 2003; accepted in final form 24 September 2003

\begin{abstract}
Sommer, Marc A. and Robert H. Wurtz. What the brain stem tells the frontal cortex. I. Oculomotor signals sent from superior colliculus to frontal eye field via mediodorsal thalamus. J Neurophysiol 91: 1381-1402, 2004.. First published October 22, 2003; First published October 22, 2003; 10.1152/jn.00738.2003. Neuronal processing in cerebral cortex and signal transmission from cortex to brain stem have been studied extensively, but little is known about the numerous feedback pathways that ascend from brain stem to cortex. In this study, we characterized the signals conveyed through an ascending pathway coursing from the superior colliculus (SC) to the frontal eye field (FEF) via mediodorsal thalamus (MD). Using antidromic and orthodromic stimulation, we identified SC source neurons, MD relay neurons, and FEF recipient neurons of the pathway in Macaca mulatta. The monkeys performed oculomotor tasks, including delayed-saccade tasks, that permitted analysis of signals such as visual activity, delay activity, and presaccadic activity. We found that the SC sends all of these signals into the pathway with no output selectivity, i.e., the signals leaving the SC resembled those found generally within the SC. Visual activity arrived in FEF too late to contribute to short-latency visual responses there, and delay activity was largely filtered out in MD. Presaccadic activity, however, seemed critical because it traveled essentially unchanged from SC to FEF. Signal transmission in the pathway was fast $(\sim 2 \mathrm{~ms}$ from $\mathrm{SC}$ to $\mathrm{FEF}$ ) and topographically organized (SC neurons drove $\mathrm{MD}$ and FEF neurons having similarly eccentric visual and movement fields). Our analysis of identified neurons in one pathway from brain stem to frontal cortex thus demonstrates that multiple signals are sent from SC to FEF with presaccadic activity being prominent. We hypothesize that a major signal conveyed by the pathway is corollary discharge information about the vector of impending saccades.
\end{abstract}

\section{N T R O D U C T I O N}

Many studies in the past half-century have characterized the signals that course from retina to cerebral cortex and then down to the brain stem motor centers that control rapid or saccadic eye movements. This work has greatly advanced our understanding of sensorimotor transformations and their attendant cognitive processes. However, there are also pathways running in the reverse direction, from brain stem up to cortex. What roles do these ascending pathways play in vision, cognition, and movement, considering that they "go against the flow" of the normal sensorimotor transformation?

Anatomical work has indicated that one such pathway in monkeys ascends from midbrain to frontal cortex via a thalamic relay (Fig. 1A). Studies showed, first, that some projections from the intermediate layers of the superior colliculus (SC) terminate at the lateral edge of the mediodorsal nucleus (MD) of the thalamus (Benevento and Fallon 1975; Harting et

Address for reprint requests and other correspondence: M. A. Sommer, Bldg 49, Room 2A50, MSC 4435, NEI, NIH, 9000 Rockville Pike, Bethesda, MD 20892-4435 (E-mail: mas@1sr.nei.nih.gov). al. 1980) and, second, that some neurons in this part of MD project to a prefrontal area important for oculomotor control, the frontal eye field (FEF) (Barbas and Mesulam 1981; Goldman-Rakic and Porrino 1985; Kievit and Kuypers 1975; Le Gros Clark and Boggon 1935; Walker 1940). Subsequent transynaptic retrograde tracing experiments using herpes virus injections in FEF found first-order labeling in thalamus, including in lateral MD, and second-order labeling in the SC intermediate layers (Lynch et al. 1994). Taken together, these results provided strong evidence for an SC-MD-FEF pathway. In the present study, we examined the signals sent through this pathway.

Our first goal was to physiologically identify neurons throughout the pathway using the classic methods of antidromic and orthodromic activation. Antidromic activation (backfiring neurons with electrical stimulation) has been employed since the earliest experiments in behaving monkeys to identify neurons projecting out of a brain area (e.g., Bizzi 1968; Evarts 1968). To identify neurons that receive input from a specific brain area, investigators have used orthodromic activation (synaptically driving neurons with stimulation) (e.g., Raybourn and Keller 1977). In the present study, we used these methods as follows. To identify SC neurons projecting into the ascending pathway, we antidromically activated them from MD. To identify FEF neurons receiving input from the pathway, we orthodromically activated them from the SC. To identify the crucial MD relay neurons linking the SC to the FEF, we orthodromically activated them from the SC and antidromically activated them from the FEF. The use of antidromic and orthodromic activation techniques yielded two further benefits as well: by analyzing activation latencies, we could determine the speed of signal transmission in the pathway, and by comparing the ability to activate MD or FEF neurons from different parts of the SC, we could establish whether neurons along the pathway were connected logically as a function of the wellknown topography in the SC (rostral SC represents small and caudal SC large saccades) (Robinson 1972).

Our second main goal was to characterize the signals conveyed through the pathway. Previous researchers hypothesized that the pathway might carry visual signals (Suzuki and Azuma 1983; Wurtz and Mohler 1976), activity related to cognitive processes (Leichnetz et al. 1981), and/or presaccadic activity (Goldberg and Bushnell 1981; Lynch et al. 1994). All of these hypotheses are plausible because recordings in the general populations of SC, MD, and FEF neurons showed that neurons in all three structures can have visual-, cognitive-, or saccade-

The costs of publication of this article were defrayed in part by the payment of page charges. The article must therefore be hereby marked "advertisement" in accordance with 18 U.S.C. Section 1734 solely to indicate this fact. 


\section{A MD Relay Neurons: Identification}
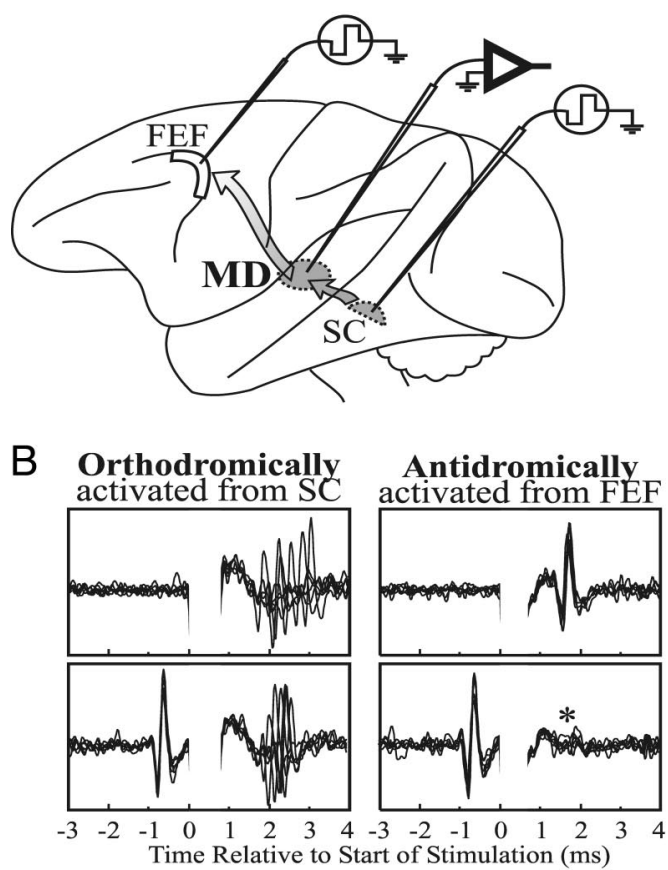

C Relay Collision Test
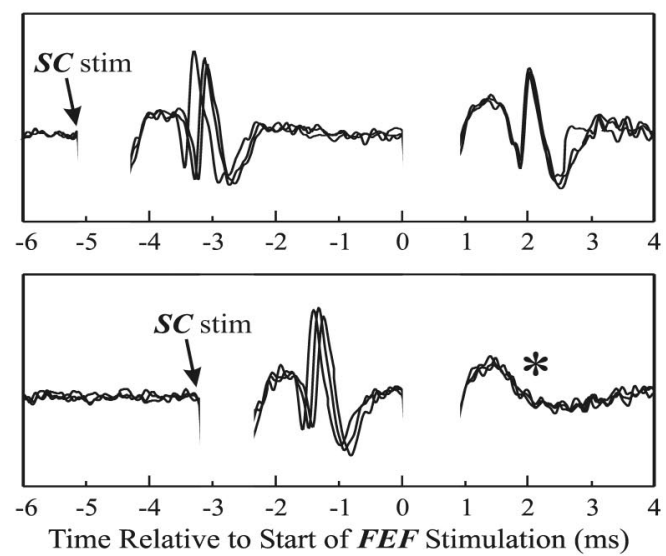

FIG. 1. Identification of mediodorsal nucleus (MD) relay neurons. A: MD relay neurons were both orthodromically activated from the superior colliculus (SC) and antidromically activated from the frontal eye field (FEF). B: action potentials from an example MD relay neuron. Left: orthodromic activation from the SC. Top: stimulation in the SC at time 0 caused the MD neuron to fire 1.5-3 ms later (several trials superimposed). Stimulus artifact was erased for clarity. Bottom: failure of the collision test; when the SC was stimulated just after a spontaneous action potential of the MD neuron, activation still occurred. Right: antidromic activation from the FEF. Top: FEF stimulation at time 0 caused the MD neuron to fire with a stable, short latency of $\sim 1.2 \mathrm{~ms}$. Bottom: success of the collision test; when the FEF was stimulated just after a spontaneous action potential of the MD neuron (within a collision interval of $\sim 1.4 \mathrm{~ms}$ ), activation failed to occur (no spikes appeared at time designated with *). $C$ : relay collision test. Time 0 is when FEF is stimulated; the time of SC stimulation varies, as labeled (SC stim). Top: the neuron was activated orthodromically from the SC, but FEF stimulation still caused antidromic activation because it occurred at sufficient delay after the orthodromically activated spikes (i.e., beyond the collision interval). Bottom: when the delay between SC and FEF stimulation was decreased to within the collision interval, the orthodromically activated spikes prevented antidromic activation (i.e., spikes are absent at time designated by *). The orthodromically and antidromically activated action potentials therefore were produced by the same neuron. related activity (FEF: reviewed by Schall 1997; MD: Schlag and Schlag-Rey 1984; Schlag-Rey and Schlag 1984; Tanibuchi and Goldman-Rakic 2003; Wyder et al. 2003; SC: reviewed by Wurtz et al. 2000). Our identification of the specific SC neurons that feed into the pathway, the specific MD neurons that serve as relays, and the specific FEF neurons that receive input from the pathway allows us to test these hypotheses by explicitly determining what signals are sent from SC to FEF and how they change along the way. To evaluate the signals, we recorded from identified neurons while monkeys performed tasks that included making delayed saccades to visual or remembered targets. We focused our analyses on visual responses, delay activity, and saccade-related activity.

We found that the pathway from SC to MD to FEF is fast conducting, topographically connected, and rich in many types of signals from purely visual to purely saccadic. The saccaderelated activity seemed especially important because unlike the other signals it coursed through the pathway with no detectable change in its strength or timing. We conclude that a major role of the pathway from SC to FEF may be to provide feedback of saccadic commands, or corollary discharge, to cerebral cortex. In the accompanying paper (Sommer and Wurtz 2004b), we describe experiments in which we tested this hypothesized function by transiently inactivating the pathway.

Some of these results were previously reported in brief articles and abstracts (Sommer and Wurtz 1998, 2000a, 2002; Wurtz and Sommer 2000).

\section{METHODS}

\section{Identifying neurons in the pathway}

The first main goal of this study was to physiologically identify neurons throughout the pathway from SC to MD to FEF. In three 5-10 kg monkeys (Macaca mulatta), we implanted scleral search coils for measuring eye position, recording cylinders for accessing the brain, and a post for immobilizing the head during experiments (see Sommer and Wurtz 2000b for details). All procedures were approved by the Institute for Animal Care and Use Committee and complied with Public Health Service Policy on the humane care and use of laboratory animals. In one monkey $(C)$, we studied identified neurons at all three levels of the pathway, in another $(B)$, we studied identified neurons in SC and MD only, and in the third $(H)$, we studied identified neurons in FEF only. Data were collected from within the left hemisphere of monkeys $C$ and $H$ and from within the right hemisphere of monkey $B$. We found the FEF and SC using recording and stimulation criteria (presaccadic activity and $<50 \mu \mathrm{A}$ current thresholds for evoking saccades) and confirmed the localizations with magnetic resonance imaging (MRI). To find MD, we used anatomical studies (Barbas and Mesulam 1981; Goldman-Rakic and Porrino 1985; Lynch et al. 1994) and stereotaxic coordinates (Martin and Bowden 1997; Olszewski 1952) as guides. We positioned MD cylinders at A8, L3 and searched in them until we found identified relay neurons (the process of identification is described next). We always inserted recording and stimulating electrodes through guide tubes (23 gauge) aimed using a grid (Crist et al. 1988) having holes $1 \mathrm{~mm}$ apart (even finer resolution was achieved in some recordings using grids with holes offset $0.5 \mathrm{~mm}$ from the normal pattern).

ANTIDROMIC AND ORTHODROMIC STIMULATION. We used standard techniques to record extracellularly from single neurons (Sommer and Wurtz 2000b). After isolating a neuron, we identified its connections using antidromic and/or orthodromic activation (for reviews, see Lemon 1984; Lipski 1981). Activation means that a neuron produced an action potential shortly after we electrically stimulated a 
distant structure (for examples see Fig. 1B, top row). Antidromic activation, or backfiring a neuron through its own axon, indicated that the neuron projected to the distant structure. Orthodromic activation, or synaptically driving the neuron, indicated that the neuron received input from the distant structure. We distinguished anti- from orthodromic activation primarily with the collision test in which electrical stimulation is timed to occur just after a spontaneous action potential of the neuron (Fig. 1B, bottom). If the neuron projects to the area containing the stimulating electrode, then the spontaneous action potential moving forward in the axon will meet the stimulationevoked action potential moving backward in the same axon, and neither will travel further because the axon will be in its absolute refractory period on both sides of the meeting point. Hence the stimulation-evoked action potential will not appear at the recording electrode (Fig. 1B, bottom right, *), whereas normally it would (Fig. $1 B$, top right). If the delay between onset of the spontaneous action potential and onset of stimulation is increased past a certain interval, $\sim 0.2-0.6 \mathrm{~ms}$ longer than the activation latency, then the collision effect ceases (not shown). If all this occurs, then the activation passes the collision test, and the neuron is considered to be antidromically activated; it projects to the distant structure holding the stimulating electrode. If the activation fails the collision test (Fig. $1 \mathrm{~B}$, compare top left and bottom left), then the neuron is considered to be orthodromically activated; it receives input from the distant structure. To complement the collision test, we also examined whether the activation latency was stable or not; stable activation latencies, exhibiting a range $\leq 0.1 \mathrm{~ms}$ (Fig. $1 B$, top right), are typical of antidromic activation, but jittery latencies, range $>0.1 \mathrm{~ms}$ (Fig. $1 B$, top left), are typical of orthodromic activation. In rare cases when we were unsure whether the activation was antidromic versus orthodromic, we abandoned the neuron and searched for a new one.

Neurons were identified as belonging to the ascending pathway according to the following criteria: SC neurons had to be antidromically activated from MD, MD neurons had to be orthodromically activated from the SC and antidromically activated from the FEF, and FEF neurons had to be orthodromically activated from the SC. To activate neurons, we stimulated through tungsten microelectrodes ( $\sim 100 \mathrm{k} \Omega$ at $1,000 \mathrm{~Hz}$; Frederick-Haer) using single, biphasic, negative-positive current pulses of $0.15 \mathrm{~ms} /$ phase. We implanted these monopolar stimulating electrodes semi-chronically for several weeks except during some experiments (as mentioned in RESULTS) in which we used moveable electrodes. Accurate placement of the stimulating electrodes was critical to this study, and therefore details about this are provided next.

SC-STIMULATING ELECTRODES. We implanted stimulating electrodes in the SC (for orthodromically activating MD or FEF neurons) with their tips in the intermediate layers in all three monkeys. We always implanted a pair of SC-stimulating electrodes with one relatively rostral (in or near the "fixation" zone; Munoz and Wurtz 1993) and one relatively caudal (typically $10-20^{\circ}$ eccentricity on the SC topographic map) (Robinson 1972), except in a few experiments when we used a single, moveable electrode to find the current threshold profile as a function of depth into the SC (e.g., Fig. $4, E$ and $F$ ). To confine stimulating currents as much as possible to the SC, we positioned stimulating electrodes on or near the representation of the horizontal meridian that runs through the center of the SC map (see Fig. $4 C, 0^{\circ}$ direction contour). Before epoxying an electrode in place, we always recorded through it to ensure that its tip was amid neurons having fixation-related, foveal visual, and/or small-saccade-related activity (for the rostral electrode) or peripheral visual responses and large-saccade-related activity (for the caudal electrode), and we stimulated through the electrode using trains of pulses to ensure that low currents $(<20 \mu \mathrm{A}$ at $350 \mathrm{~Hz}$ for $70 \mathrm{~ms}$ using biphasic pulses having durations of $0.25 \mathrm{~ms} /$ phase) fixed the eyes in place or evoked small saccades (for the rostral electrode) or evoked larger saccades (for the caudal electrode). After several weeks, the implanted electrodes be- came unusable (they failed to pass current reliably), at which time we replaced them with new electrodes at slightly different SC locations. Over the course of the study, we used a total of 10 SC electrode pairs in the three monkeys. On average, we placed rostral electrode tips at $1.7^{\circ}$ eccentricity on the SC map and $1.8 \mathrm{~mm}$ depth below the SC surface and caudal electrode tips at $13.0^{\circ}$ eccentricity and $1.7 \mathrm{~mm}$ depth. The current thresholds for orthodromically activating neurons using these SC electrodes were on average $264 \mu \mathrm{A}$ (range: 7-718 $\mu \mathrm{A}$, $n=45$ ) for MD neurons and $110 \mu \mathrm{A}$ (range: $16-540 \mu \mathrm{A}, n=47$ ) for FEF neurons. The difference in these averages probably was not important; it may have been an artifact of slightly different SC stimulating electrode placements in each of the three monkeys.

FEF-STIMULATING ELECTRODES. We implanted stimulating electrodes in the FEF (for antidromically activating MD neurons) in two monkeys, using slightly different methods to place them in each animal. In one monkey, we positioned an array of three electrodes with their tips at the sites of FEF neurons that we previously found to be activated orthodromically from the SC; that is, the electrodes were positioned as closely as possible to the target zone of the ascending pathway. In the other monkey, we simply positioned arrays of three or four electrodes with their tips in the middle layers of the FEF as indicated by recordings and MRI. Both methods were successful, although the former method was superior in that it yielded markedly lower current thresholds for antidromically activating MD neurons (average: $317 \mu \mathrm{A}$, range: $43-836 \mu \mathrm{A}, n=30$ neurons) as compared with the latter method (average: $1,298 \mu \mathrm{A}$, range: $335-1,850 \mu \mathrm{A}, n=$ 16 neurons). Although current thresholds using the latter method were relatively high, it is unlikely that the stimulation activated axons outside of the FEF. Using the relation distance $=(\text { current } / K)^{0.5}$ (Tehovnik 1996), the average threshold of $1,298 \mu \mathrm{A}$ at a pulse duration of $0.15 \mathrm{~ms}$ should activate low-threshold axons $(K \approx 381$ $\left.\mu \mathrm{A} / \mathrm{mm}^{2}\right)$ only within $1.8 \mathrm{~mm}$ and high-threshold axons $(K \approx 4,844$ $\mu \mathrm{A} / \mathrm{mm}^{2}$ ) only within $0.5 \mathrm{~mm}$ of the electrode tip.

MD-STIMULATING ELECTRODES. We implanted stimulating electrodes in MD (for antidromically activating SC neurons) in two monkeys at sites of previously recorded MD relay neurons. To minimize damage to the relay neurons and permit further experiments in the region (i.e., reversible inactivations; Sommer and Wurtz 2004b), we used only a single stimulating electrode in the MD of each monkey. We placed it at the most posterior site found to contain relay neurons, reasoning that SC afferents follow a posterior-anterior trajectory so that most should pass near this electrode before terminating in MD. Current thresholds for antidromically activating SC neurons from MD were on average $270 \mu \mathrm{A}$ (range: $35-1,284 \mu \mathrm{A}, n=48$ ).

TOPOGRAPHY OF PROJECTIONS. We also used orthodromic activation to search for projection topographies in the pathway. The concept was to see if stimulation in rostral (or caudal) SC preferentially drove $\mathrm{MD}$ or FEF neurons that represented small (or large) saccades as would be logical according to the SC map (Robinson 1972). This required measuring the visual and/or movement field of each MD or FEF neuron, and our procedure for doing this is described in the VISUAL AND MOVEMENT FIELDS section. We also had to estimate what part of the SC provided input to the neuron, and to do this, we calculated for each MD or FEF neuron a contrast ratio called the electrode preference index (EPI). The principle behind the EPI was as follows. Because we typically used two stimulating electrodes in the $\mathrm{SC}$, one rostral and one caudal, we could compare the ability to drive an MD or FEF neuron from rostral versus caudal SC. If stimulation through one electrode activated the MD or FEF neuron at a lower current threshold than did stimulation through the other, then the former, "better" electrode was probably located nearer to those SC neurons that drove the MD or FEF neuron. EPI $=\left(I_{\mathrm{r}}-I_{\mathrm{c}}\right) /\left(I_{\mathrm{r}}+I_{\mathrm{c}}\right)$, where $I_{\mathrm{r}}$ and $I_{\mathrm{c}}$ were the current thresholds for activating the recorded neuron from rostral and caudal SC (threshold was defined as the current causing activation in $50 \%$ of trials). An EPI closer to -1 or +1 
suggested that the neuron was preferentially driven from projections originating closer to rostral or caudal SC, respectively.

\section{Characterizing signals in the pathway}

Our second main goal was to evaluate the signals sent from SC to MD to FEF. After physiologically identifying a neuron as belonging to the pathway as described in the preceding text, we analyzed its signals by having the monkey perform a series of tasks. Details of the testing apparatus were described previously (Sommer and Wurtz 2000b). Briefly, the monkey faced a tangent screen on which visual stimuli were projected by an LCD monitor. Visual stimuli were $0.3 \times$ $0.3^{\circ}$ blue or red spots $\left(0.6 \mathrm{~cd} / \mathrm{m}^{2}\right)$ presented on a dark background $(0.1$ $\mathrm{cd} / \mathrm{m}^{2}$ ) with dim ambient room light. Personal computers controlled the presentation of visual stimuli and recorded at $1 \mathrm{kHz}$ the eye position, the occurrence of action potentials, and the timing of task events.

DELAYED-SACCADE TASKS. The purpose of these tasks was to search for visual responses, presaccadic activity, and tonic activity known as delay activity that seems related to cognitive processes such as memory or planning (e.g., see Sommer and Wurtz 2001). The delayed-saccade tasks are diagrammed in Fig. 10A. All trials began with a fixation point appearing in the center of the screen. After the monkey foveated it for 500-800 ms (pseudorandomly varied, like all task timings in this study), a target appeared at the estimated center of the visual and/or movement field. In visual trials of the task (Fig. 10A, Vis.), the target remained lit for the rest of the trial; in memory trials (Fig. 10A, Mem.), the target disappeared after $100 \mathrm{~ms}$. In all trials, after a delay period of $500-1,000 \mathrm{~ms}$ the fixation spot disappeared, cueing the monkey to make a saccade to the location of the target after which a water reward was delivered.

To analyze the data, we quantified and compared the mean firing rates during five periods (Fig. 10A, analysis epochs). The baseline epoch spanned 500-200 ms before target onset, the visual epoch $50-150 \mathrm{~ms}$ after target onset, the delay period epoch $300-0 \mathrm{~ms}$ before the cue to move, the presaccadic epoch 50-0 ms before saccade initiation, and the postsaccadic epoch $50-150 \mathrm{~ms}$ after saccade termination. To see if the neuronal activity changed at all during the task, we ran an ANOVA on the data. If significant (at $P<0.01$ ), we performed an all-pairwise multiple comparison test (Student-Newman Keuls or Dunn's) to reveal whether firing rates in specific pairs of epochs differed from each other (at $P<0.05$ ). We defined various types of signals according to comparisons between epochs, as follows. A phasic visual response occurred if the visual epoch activity exceeded the baseline epoch activity in either visual or memory trials. A tonic visual response occurred if the activity in the delay period of visual trials, during which time the receptive field was steadily illuminated, exceeded both the baseline activity in visual trials and the delay period activity in memory trials, two periods during which no visual stimuli were in the receptive field. Delay activity occurred if the delay period activity in memory trials exceeded the baseline activity in those trials; because the physical characteristics (fixation spot on, no peripheral visual stimulus) and the motor state (steady fixation) were identical in both of these epochs, differing activity between them must represent differences in the internal state of the animal presumably related to memory or other cognitive processes. Presaccadic activity occurred if presaccadic epoch activity exceeded both the delay period and the baseline activity in either visual or memory trials. Postsaccadic activity occurred if the postsaccadic epoch activity exceeded both the presaccadic and baseline epoch activities. We also performed other analyses on the firing rate data, such as determination of visual response latency, as will be described in the RESULTS.

GAP TASK. The purpose of this task was to look for "gap activity" during a brief period (the gap) after a fixation spot disappears but before a saccadic target appears. Such activity may be related to cognitive processes such as disengaging fixation or preparing to make a saccade (e.g., Dias and Bruce 1994; Munoz et al. 2000). In the gap task (Fig. 12A), the monkey fixated a spot for 500-800 ms and then the spot disappeared; the monkey had to maintain fixation on the blank screen, and then, after a 200-ms gap period, a target was presented at the estimated center of the visual and/or movement field; the monkey then looked at the target to receive its reward. We compared the mean firing rate during the gap period epoch, from 50 $\mathrm{ms}$ before target onset to $50 \mathrm{~ms}$ after (Fig. 12A), with the firing rate during a baseline epoch 500-200 ms before start of the gap. Gap activity occurred if the gap period activity exceeded the baseline activity.

FIXATION BLINK TASK. The purpose of this task was to quantify foveal visual receptive fields and fixation-related activity in neurons that qualitatively appeared to exhibit these characteristics during the delayed-saccade and gap tasks. The task was very simple (diagram not shown): the monkey foveated a spot for $500-1,000 \mathrm{~ms}$, the spot disappeared for $400-600 \mathrm{~ms}$, and then the spot reappeared at the same place for 500-1,000 ms. The monkey was rewarded if it steadily fixated throughout the trial. We used three analysis epochs: a baseline epoch spanning 300-0 ms before fixation spot onset, a fixation epoch during the temporary disappearance of the fixation spot $(300-0 \mathrm{~ms}$ before it reappeared), and a visual epoch just after the visual stimulus was flashed onto the fovea (100-300 ms after fixation spot reappearance). We compared the mean firing rates during these epochs using ANOVA and multiple comparison tests as described in the preceding text for the delayed-saccade task. A neuron carried a fixation signal if its activity in the fixation epoch was different from the baseline epoch activity and a foveal visual response if its activity in the visual epoch exceeded both the fixation and baseline epoch activities.

VISUAL AND MOVEMENT FIELDS. For every identified neuron, we measured the range of locations where visual stimuli caused it to fire (its visual field) and the range of saccadic vectors for which it fired presaccadically (its movement field). As with all testing in this study, we performed these measurements while the monkey's head was held stationary. We first estimated these fields on-line (see Fig. 7A, pink) by having the monkey make saccades to a variety of target locations while we inspected rasters of neuronal activity. For neurons having both visual and movement fields, it was clear by inspection that the fields were always highly coincident, so a single estimated field accurately represented both component fields. From this initial testing, we found the location of the target that evoked maximal activity, and we presented targets at this estimated best target location during the delayed-saccade and gap tasks described in the preceding text.

To permit off-line quantitative analysis of the visual and movement fields, we collected two data files while the monkey performed a visually guided saccade task in which it fixated a spot for 500-800 ms that then disappeared just as a peripheral target appeared. The monkey had to make a saccade to the target to receive a reward. First, we randomly presented targets in a direction series of eight possible locations around a circle having its radius equal to the estimated best eccentricity (Fig. $7 A$, orange). We set the directions to every $45^{\circ}$ in angle starting from horizontal. Second, we randomly presented targets in an eccentricity series of eight possible locations along a line oriented at the estimated best direction (Fig. $7 A$, blue). We set the eccentricities to an array of $\left[2.5,5,10,20,30,40,50,60^{\circ}\right]$ or adjusted them to better match the estimated range of the field(s) by multiplying this array by a scalar (e.g., by 0.5 for fields near the fovea). To optimize the accuracy of the eye position measurements, we arranged the fixation point and target so that most saccades in the eccentricity series traveled symmetrically across the center of the screen (Aizawa and Wurtz 1998; Munoz and Wurtz 1995), e.g., $20^{\circ}$ leftward saccades were elicited by placing the fixation spot $10^{\circ}$ right of center and the saccadic target $10^{\circ}$ left of center. We did this for all target eccentricities except for the two smallest for which we used a central fixation spot.

For every neuron having a significant visual response (as deter- 
mined quantitatively using the delayed-saccade tasks), we reconstructed its visual field off-line by measuring average visual activity 50-150 ms after target onset in the direction and eccentricity series data. Similarly, for every neuron having significant presaccadic activity, we reconstructed its movement field by measuring average presaccadic activity $50-0 \mathrm{~ms}$ before the saccade in the direction and eccentricity series data. We fitted Gaussians to the direction series data (least-squares approximation) separately for the visual activity and the presaccadic activity, yielding direction cross-sections through the visual and movement fields (Fig. 7, $B$ and $C$, left). We measured the direction range as the range of directions for which the Gaussian was $>2$ SDs above the mean baseline firing rate (we measured baselines 300-0 ms before target onset). The direction series data were shifted before fitting the Gaussian so that the fitted curve would peak near the center of the range; periodic curve fits, e.g., cosines, were attempted but the fields were too narrow to be well fit by them. We fitted splines to the eccentricity series data (Munoz and Wurtz 1995), yielding eccentricity cross-sections through the visual and movement fields (Fig. 7, $B$ and $C$, right). We measured the eccentricity range as the range of eccentricities for which the spline was $>2$ SDs above the mean baseline firing rate. We used cubic spline fits except when they were clearly inadequate due to sharply varying data points (e.g., Fig. 7D, left and middle), in which case we used a ninth-order spline. We performed all curve fitting with Matlab (The MathWorks).

We note that for quantifying movement fields, activity was plotted against the actual direction or amplitude of each saccade, which is why data clusters in Fig. 7, $C$ and $D$, exhibit scatter in both the $x$ - and y-axis directions. For movement field eccentricity, therefore, spline fits were least-squares approximations. For visual fields, in contrast, we calculated the mean firing rate for each target location, which is why there is a single data point at each direction or eccentricity in Fig. $7 B$. Also, for simplicity we use the term "eccentricity" to describe radial extent for visual and movement fields, although "amplitude" would be more precise for movement fields.

DETECTION OF SACCADES AND FIXATIONS. On-line, we identified saccades and fixations using real-time detection software written in-house. A saccade was accurate if it landed in a rectangular virtual window surrounding the target location that ranged in size from $1^{\circ}$ horizontally $\times 2^{\circ}$ vertically for targets at small eccentricity (e.g., $4^{\circ}$ ) to $10 \times 20^{\circ}$ for targets located at large eccentricity (e.g., $40^{\circ}$ ). Windows for enforcing fixation were $2 \times 2^{\circ}$ squares around the fixation point. Off-line, we used software running a template-matching algorithm to automatically detect saccades in eye-position records. We verified the accuracy of saccade detection by visually inspecting the data from every trial.

STATISTICS. Unless explicitly noted otherwise, throughout this paper we compare data sets using Student's $t$-test, if judged normal by the Kolmogorov-Smirnov test and of equal variance by the Levene Median test, or else the Mann-Whitney rank sum test, and we analyze correlations using Pearson's test.

\section{RES U L T S}

\section{Identified neurons in the ascending pathway}

We studied a total of 151 identified neurons: 48 SC source neurons projecting into the ascending pathway, $47 \mathrm{MD}$ relay neurons linking $\mathrm{SC}$ to $\mathrm{FEF}$, and $56 \mathrm{FEF}$ recipient neurons targeted by the pathway.

MD RELAY NEURONS. Identification of MD relay neurons was the crucial first step in our study because it explicitly confirmed the presence of a pathway from SC to MD to FEF. Every time we isolated a neuron in $\mathrm{MD}$, we tried to activate it using stimulating electrodes in the SC and the FEF (Fig. 1A). All 47
MD relay neurons in our sample (17 from monkey $B, 30$ from monkey $C$ ) were orthodromically activated from the SC as evidenced by activations that always failed the collision test (Fig. 1B, bottom left) and stimulation-evoked action potentials that nearly always had jittery latencies (Fig. $1 B$, top and bottom left). All the MD neurons also were antidromically activated from the FEF, as evidenced by activations that always passed the collision test (Fig. 1B, bottom right) and stimulationevoked action potentials that always had stable latencies (Fig. $1 B$, top right). These individual MD neurons therefore received $\mathrm{SC}$ input and projected to the FEF, verifying the existence of a pathway from SC to MD to FEF.

We recorded only from well-isolated MD neurons and were always confident that both the orthodromically and antidromically activated action potentials were produced by the same neuron. To confirm this, when time permitted we also used the relay collision test (Fig. $1 C$ ), which has been used previously for identifying thalamic relay neurons of other pathways (e.g., Deschênes et al. 1982; Zhu and Lo 1998). Instead of waiting for the MD neuron to fire spontaneously to perform the collision test, we made it fire by orthodromically activating it from the SC. The orthodromically activated action potential could annihilate an action potential evoked antidromically from the FEF only if both sets of action potentials were produced by the same MD neuron. This always occurred; every neuron tested $(11 / 11)$ passed the relay collision test.

In both monkeys, we found $\mathrm{MD}$ relay neurons $\sim 3 \mathrm{~mm}$ lateral of the midline and $\sim 7-9 \mathrm{~mm}$ anterior to the interaural line (Fig. 2A). In monkey $B$, we took particular care to search outside this narrow zone but found no other relay neurons (Fig. $2 A$, right). Many of the surrounding neurons could be orthodromically activated from the SC or antidromically activated from the FEF but not both (Fig. 2B); we do not know to where these SC-recipient neurons projected or from where these FEF-projecting neurons received their input, and we did not study their signals. We found MD relay neurons $\sim 18-23 \mathrm{~mm}$ below the top of the brain, slightly deeper (by $\sim 3 \mathrm{~mm}$ ) in one monkey than in the other (Fig. $2 C$ ). The coordinates of the MD relay neurons corresponded well with the lateral edge of MD as described in stereotaxic atlases (Martin and Bowden 1997; Olszewski 1952) and as predicted by anatomical studies (Barbas and Mesulam 1981; Goldman-Rakic and Porrino 1985; Lynch et al. 1994). This lateral MD location was histologically verified in both monkeys (Fig. 3 shows the results from monkey $C$ ).

SC SOURCE NEURONS. After collecting the sample of MD relay neurons in each monkey, we implanted a stimulating electrode with its tip amid these neurons and began recording in the SC (Fig. 4A). All 48 SC source neurons (33 from monkey B, 15 from monkey $C$ ) were antidromically activated from the MD electrode (Fig. 4B). We found source neurons in every site that we explored over a large range of the rostrocaudal and mediolateral extent of the SC (Fig. 4C). In each penetration, we started looking for SC source neurons at the top of the superficial layers and continued searching until we left the SC $\sim 4-5$ $\mathrm{mm}$ below. We found source neurons primarily 1-3 mm below the surface (Fig. 4D), corresponding approximately to the intermediate layers. To test this assessment that SC intermediate layer neurons drove the MD relay neurons, we also measured the optimal depths of stimulation within the SC for 
A MD Relay Neurons: Location

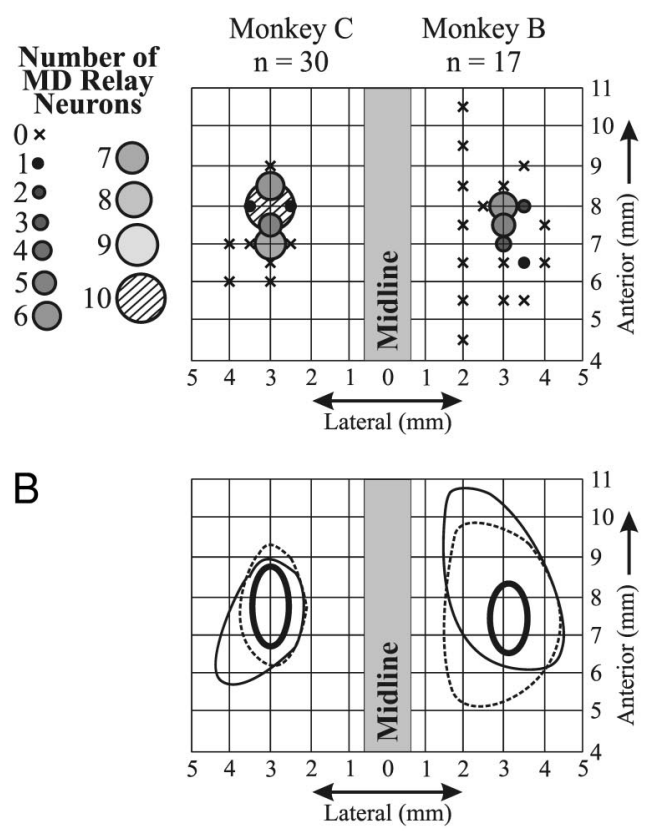

Anti from FEF and ortho from SC (relay neurons) Ortho from SC (but not anti from FEF) Anti from FEF (but not ortho from SC)

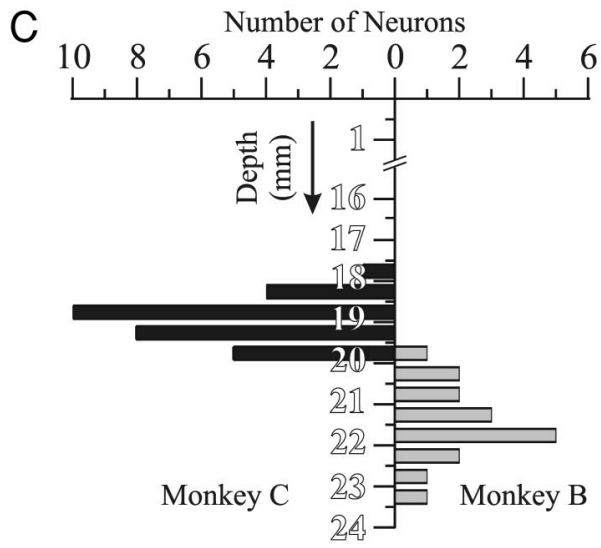

FIG. 2. Locations of MD relay neurons. $A$ : all recording sites in monkeys $C$ (left) and $B(r i g h t)$. Legend at left shows number of relay neurons found at each site. "Anterior" locations are stereotaxic AP coordinates, relative to interaural line. $B$ : boundaries showing approximate ranges of relay neurons (activated from both SC and FEF) as compared with broader ranges of neurons activated from either SC or FEF but not both (legend at bottom). $C$ : depths of relay neurons relative to the top of the brain.

orthodromically activating MD relay neurons. We did this for eight MD relay neurons by moving a stimulating electrode through the SC and finding the depth of lowest current threshold for activation. Figure $4 E$ shows a current threshold profile for one experiment: the minimum was in or near the intermediate layers where we found strong visual- and saccade-related activity during recordings in the same penetration. In most cases (7/8), threshold minima were in the intermediate layers (Fig. $4 F$ ), whereas in one case, the threshold minimum was deeper. We never found a threshold minimum in the purely visual superficial layers. These current threshold minima correspond well with the depths of the SC source neurons (cf. Fig. $4 D$ ), and therefore both lines of evidence agree that SC input to
MD Relay Neurons: Histology

A

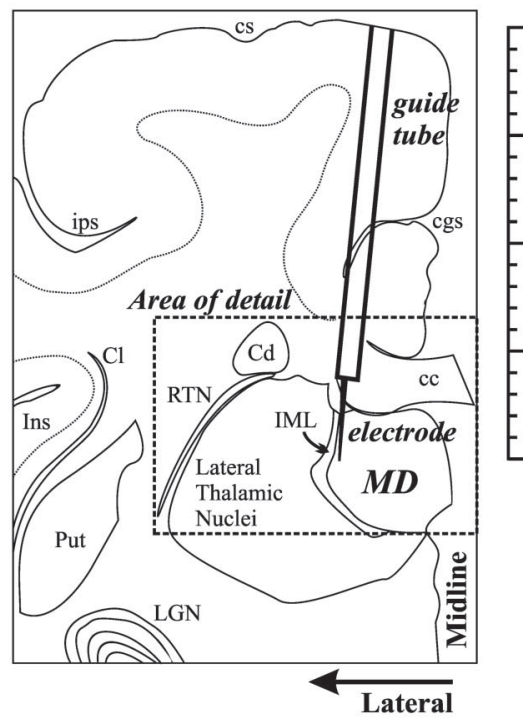

B Cell Body Stain

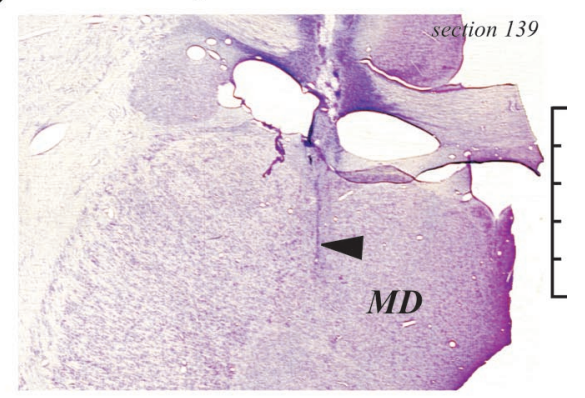

C Myelin Stain

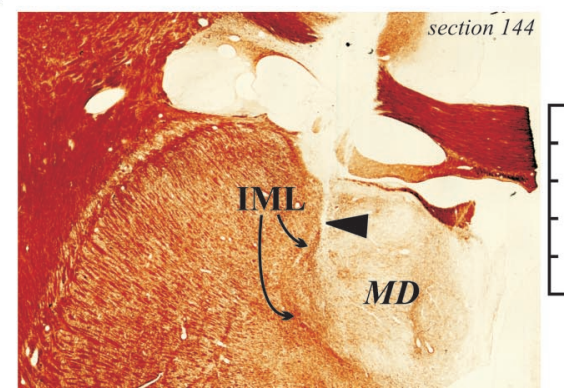

FIG. 3. Histological recovery of penetrations into the lateral edge of MD in monkey $C$. Sections (50 $\mu$ thick) were cut coronally and alternately stained for cell bodies (thionin) and myelin (modified protocol of Gallyas 1979). Scale bars are shown at right in all panels $(1 \mathrm{~mm} / \mathrm{tick}) . A$ : a sketch of the general region. Guide tubes pierced the corpus collosum (cc) and electrodes entered the lateral edge of MD, just medial to the internal medullary lamina (IML). $B$ : cell body stain, magnified from the area of detail in $A$. The black arrowhead points to a prominent penetration that yielded numerous relay neurons. $C$ : myelin stain of the same area (from a nearby section), in which the IML can be seen along with the penetration (black arrowhead) just medial to it, i.e., at the lateral edge of MD. In both $B$ and $C$, damage from the guide tube can be seen above the arrowhead in the corpus collosum. $\mathrm{Cd}$, caudate nucleus; cgs, cingulate sulcus; $\mathrm{Cl}$, claustrum; cs, central sulcus; Ins, insula; ips, intraparietal sulcus; LGN, lateral geniculate nucleus; Put, putamen; RTN, reticular nucleus of the thalamus. The region labeled lateral thalamic nuclei probably consisted of the ventrolateral and ventroposterolateral nuclei (see Olszewski 1952), but we did not attempt to verify this anatomically. 
A SC Source Neurons: Identification

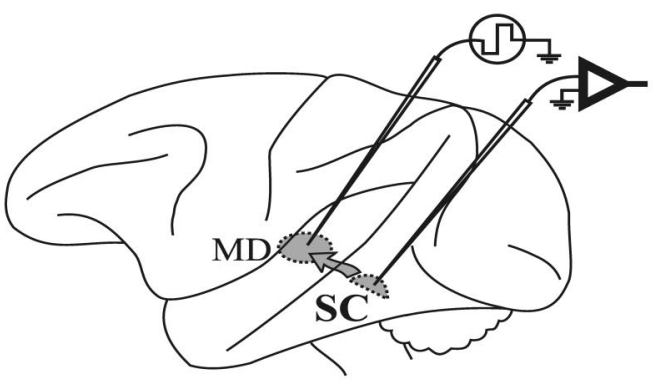

C

SC Source Neurons: Location

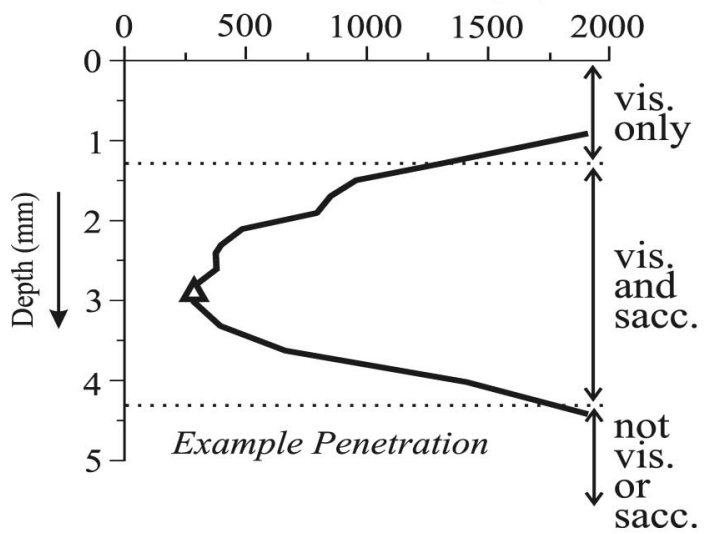

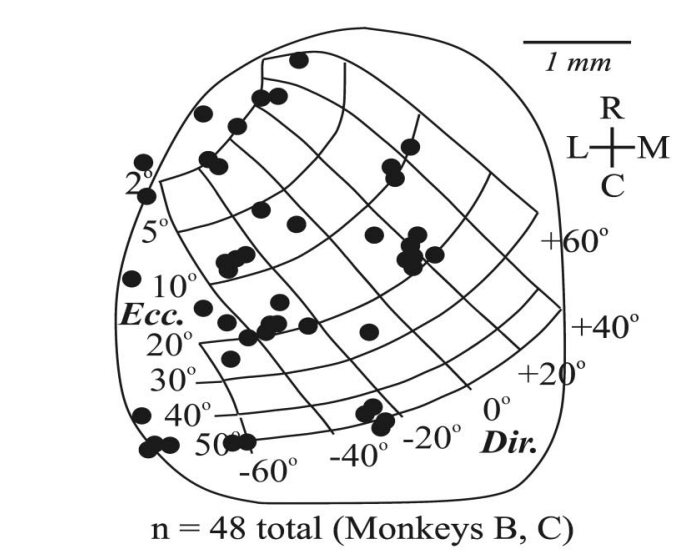

$E$

Current Threshold $(\mu \mathrm{A})$

sacc.
B

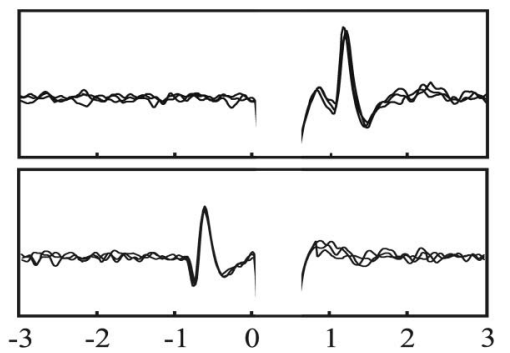

Time Relative to Start of $\boldsymbol{M D}$ Stimulation (ms)
$\mathrm{D}$

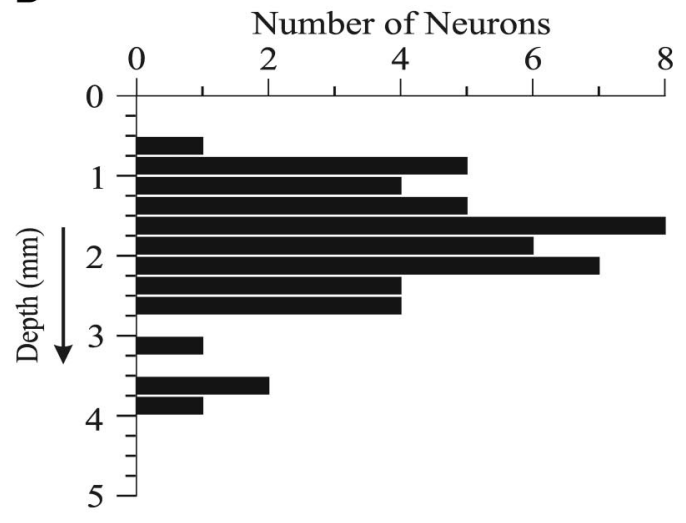

$\mathrm{F}$

Current Threshold $(\mu \mathrm{A})$

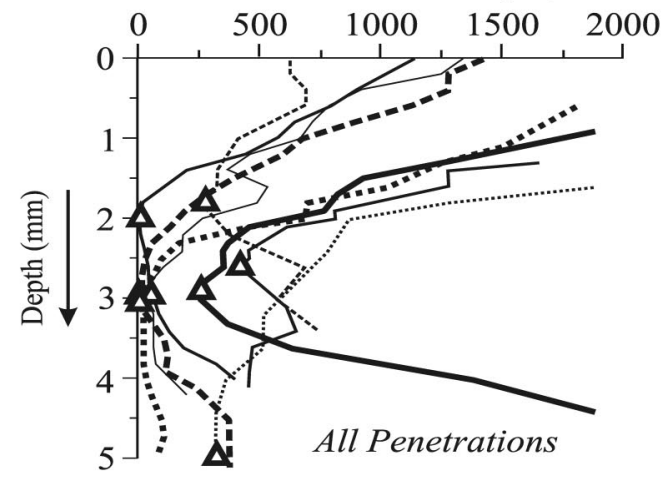

FIG. 4. Identification and location of SC source neurons. A: all of the SC source neurons were antidromically activated from sites of previously recorded MD relay neurons. $B$ : action potentials from a SC source neuron. Top: MD stimulation at time 0 caused the SC neuron to fire with a stable, short latency of $\sim 0.8 \mathrm{~ms}$. Bottom: success of the collision test. $C$ : mediolateral and rostrocaudal locations of the neurons. Using information from the visual field and/or movement field of each SC neuron, along with results of electrically evoking saccades, we estimated all the neuron locations (•) on the standard SC map of Robinson (1972). Ecc., eccentricity (size of electrically evoked saccades increases along this axis); Dir., direction (angle of electrically evoked saccades changes along this axis, with positive angles upward and negative angles downward). R, rostral; C, caudal; M, medial; L, lateral. Some sites fall slightly outside of Robinson's map, e.g., those representing saccades with more of a downward direction than he tested (directions from -60 to $-90^{\circ}$ ). Data from monkey $B$ were collected from the right SC but for simplicity are represented here on the left SC for combination with data from monkey $C$. D: depths of the neurons relative to the top of the SC. E: for comparison with neuronal depth data in $D$, an example current threshold profile for orthodromically activating an MD relay neuron is shown. Current threshold (abscissa) is plotted against the depth of the stimulating electrode tip in the SC (ordinate). Recordings through the stimulating electrode revealed the depth range where neurons had only visual responses ("vis. only" region, i.e., the superficial layers) and the depth range where neurons had visual- and saccade-related activity ("vis. and sacc." region, i.e., the intermediate and deep layers). Just below the SC, neurons had no visual- or saccade-related activity ("not vis. or sacc." region). The current threshold minimum $(\triangle)$ was $265 \mu \mathrm{A}$, at $2.8 \mathrm{~mm}$ deep. $F$ : results of all 8 tests in which the current threshold for driving MD relay neurons was evaluated as a function of electrode tip depth in the SC. 
the MD relay neurons arises primarily from the intermediate layers.

FEF RECIPIENT NEURONS. All 56 FEF recipient neurons (13 from monkey $C, 43$ from monkey $H$ ) were orthodromically activated from the SC (Fig. 5, $A$ and $B$ ), and, as we previously reported, current thresholds for driving them were lowest in the SC intermediate layers (Sommer and Wurtz 1998). Note that it was crucial to activate FEF recipient neurons from the $\mathrm{SC}$, not from $\mathrm{MD}$; as was shown in Fig. $2 B$ (thin, solid-line boundaries), many MD neurons that project to the FEF do not seem to get input from the SC, and therefore stimulating MD instead of SC could mistakenly identify FEF neurons that are targeted by pathways other than that originating in the SC. Recipient neurons were located throughout most of the mediolateral range of the FEF (Fig. 5C) and from near the top of cortex to $\sim 9 \mathrm{~mm}$ deep (as the electrode traversed the anterior bank of the arcuate sulcus; Fig. 5D). We often found FEF recipient neurons in the same penetrations that yielded FEF SC-projecting neurons (Sommer and Wurtz 2000b), but these two classes of neurons were not intermingled; they were always separated from each other by at least a few hundred microns in depth. The FEF recipient neurons were likely in layer IV, where most thalamic afferents terminate (Giguere and Goldman-Rakic
1988). FEF SC-projecting neurons are found only in layer V (Fries 1984; Leichnetz et al. 1981).

SIGNAL SPEED IN THE PATHWAY. Activation latencies, the delays from stimulation onset to the earliest action potentials evoked in activated neurons, were very brief. SC to MD antidromic latencies (Fig. 6A, shaded histogram) had a median of $0.83 \mathrm{~ms}$ and $\mathrm{MD}$ to $\mathrm{FEF}$ antidromic latencies (inverted histogram) had a median of $1.0 \mathrm{~ms}$. These two distributions were not significantly different $(P>0.05)$. SC to MD orthodromic activation latencies (dashed-line histogram) had a median of $1.4 \mathrm{~ms}$, significantly longer than the antidromic activation latency for the same projection (cf. "SC to MD anti" histogram). The additional time $(0.57 \mathrm{~ms})$ was presumably the SC-MD synaptic delay.

The SC to FEF orthodromic latencies are shown in Fig. $6 B$ (dashed-line histogram); their median was $2.2 \mathrm{~ms}$. Consistent with our assumption that the FEF recipient neurons were driven from the $\mathrm{SC}$ via MD relay neurons, this distribution of $\mathrm{SC}$ to FEF orthodromic latencies was not significantly different $(P>0.05)$ from the predicted distribution of the transmission time through the SC-MD-FEF pathway (Fig. 6B, shaded histogram), which had a median of $2.4 \mathrm{~ms}$. The predicted distribution was derived by adding the time it takes for action

\section{A FEF Recipient Neurons: Identification}

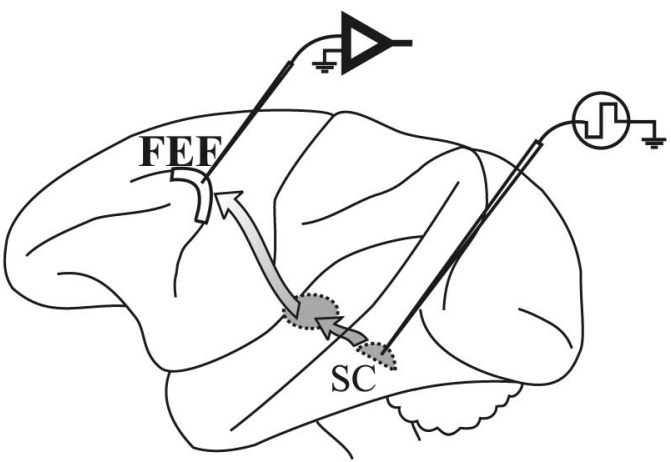

B

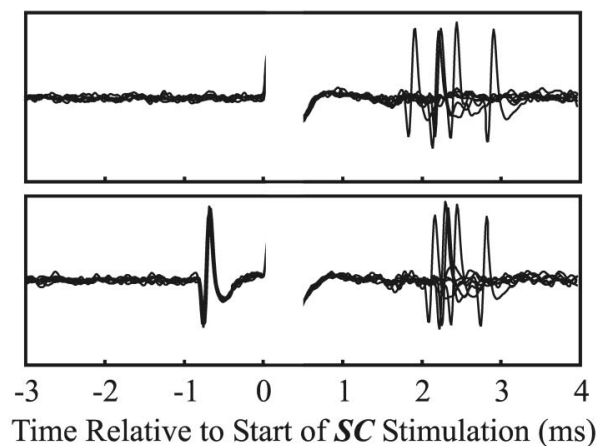

C FEF Recipient Neurons: Location

D
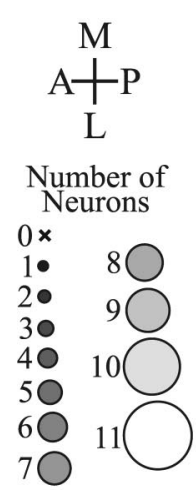
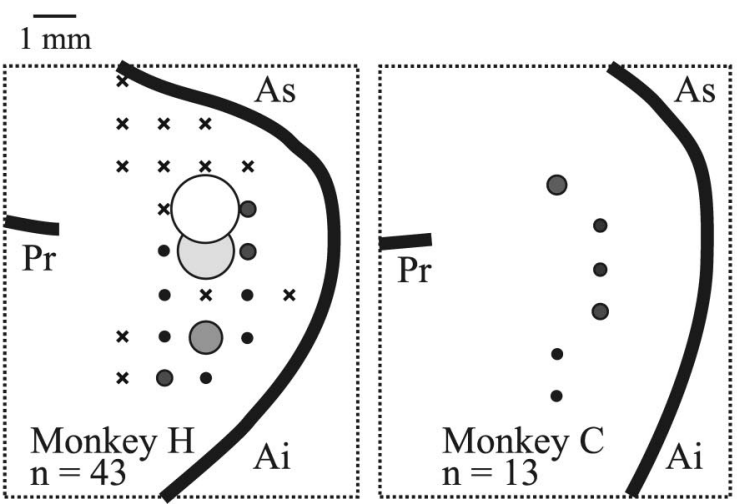

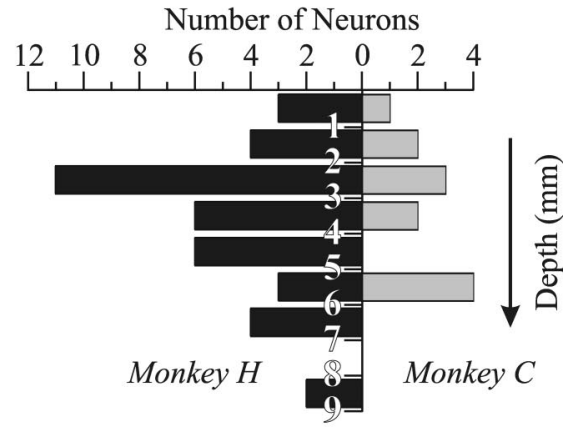

FIG. 5. Identification and location of FEF recipient neurons. $A$ : all of these neurons were orthodromically activated from the SC. $B$ : action potentials from an FEF recipient neuron. Top: SC stimulation at time 0 caused the FEF neuron to fire with a jittery latency from $\sim 1.7$ to $2.7 \mathrm{~ms}$. Bottom: failure of the collision test. $C$ : mediolateral and anterioposterior locations of the neurons. Penetration sites yielding these neurons are shown relative to the arcuate sulcus (As, its superior limb; Ai, its inferior limb) and the posterior tip of the principal sulcus (Pr). Sulcal locations were determined by inspection during surgery, MRI, and for monkey $H$, histology. A, anterior; P, posterior. $D$ : depths of the neurons. 

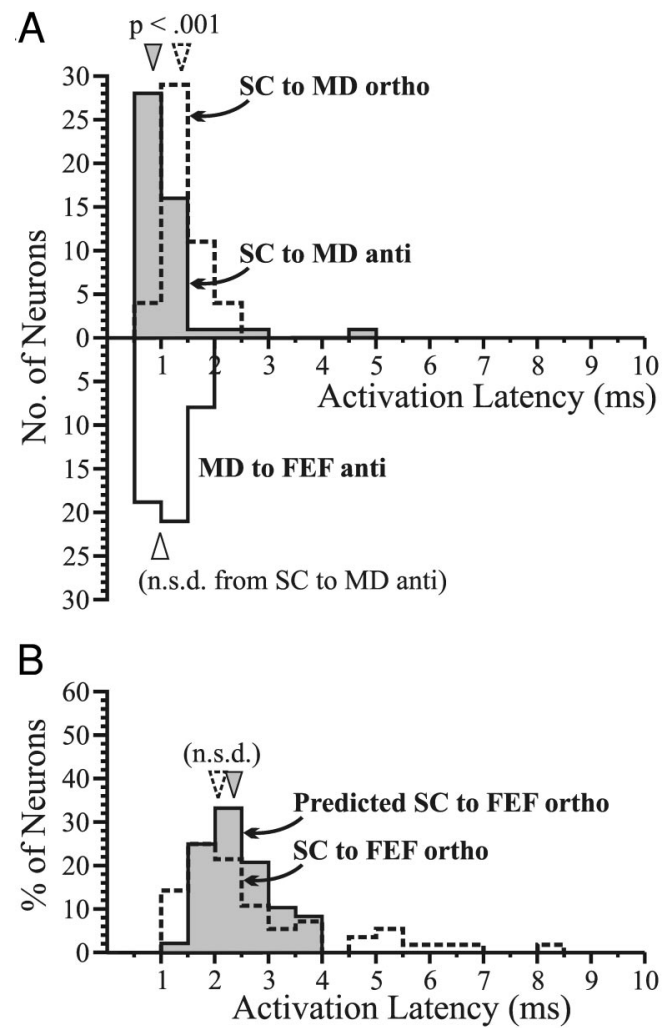

FIG. 6. Activation latencies of the neurons. A, upright histograms: for the $\mathrm{SC}$ to MD projection, the distributions of antidromic activation latencies obtained when stimulating MD and recording from SC ("SC to MD anti") and orthodromic activation latencies obtained when stimulating SC and recording from MD ("SC to MD ortho") are shown. Inverted histogram: for the MD to FEF projection, the distribution of antidromic activation latencies is shown ("MD to FEF anti"). Arrowheads show median latencies, and statistical results are indicated. $B$ : the actual distribution of orthodromic latencies from SC to FEF ("SC to FEF ortho") compared with the predicted distribution found by summing the orthodromic activation latency from SC to MD with the antidromic activation latency from MD to FEF for every MD relay neuron ("Predicted SC to FEF ortho"). The distributions were not significantly different, n.s.d.

potentials to travel from SC to MD (SC to MD orthodromic latency) to the time it takes for action potentials to travel from MD to FEF (MD to FEF antidromic latency) for each MD relay neuron. This distribution might be made more accurate by adding in the MD to FEF synaptic delay and subtracting out the FEF utilization time (the time it takes for stimulation to evoke an action potential), but these values are not known.

PROJECTION TOPOGRAPHY IN THE PATHWAY. We also used our activation methods to examine whether neurons in the pathway were linked together in a logical manner according to the topography of the SC. We expected MD and FEF neurons having small eccentricity fields to receive input primarily from rostral SC and those having large eccentricity fields to receive input primarily from caudal SC (see Fig. $4 C$ for SC topography) (Robinson 1972). First we document the general characteristics of visual and movement fields throughout the pathway, and then we analyze the projection topography.

Figure $7, A-C$, summarizes how we measured the visual and movement fields for one example MD relay neuron (see METHODS for details). It was clear from initial testing that the neuron had both visual- and saccade-related activity and that its visual and movement fields were almost perfectly coincident. After estimating the average location and size of the fields (Fig. 7A, pink), we had the monkey make saccades to targets in a direction series (Fig. 7A, orange) and eccentricity series (Fig. $7 A$, blue) that cut through the estimated fields. We plotted visual responses as a function of direction (Fig. 7B, left) and eccentricity (Fig. 7B, right) to reconstruct the visual field and similarly plotted presaccadic activity to reconstruct the movement field (Fig. 7C). This example neuron had a visual field (Fig. $7 B$ ) with best direction $35^{\circ}$, direction range $90^{\circ}$, best eccentricity $16^{\circ}$, and eccentricity range $37^{\circ}$. Its movement field was nearly identical (Fig. $7 \mathrm{C}$ ), having a best direction of $27^{\circ}$, direction range of $128^{\circ}$, best eccentricity of $16^{\circ}$, and eccentricity range of $31^{\circ}$. The correspondence of this neuron's visual and movement fields was typical; similar overlap was seen in all of our MD, SC, and FEF neurons that had both visual and presaccadic activity.

The visual and movement fields for this example neuron were "closed", i.e., bounded in eccentricity (Fig. 7, $B$ and $C$, right), but some other neurons had "open" visual or movement fields in that visual or presaccadic activity was significantly elevated even at the furthest eccentricity tested. Open fields have been described previously for SC and FEF neurons (Bruce and Goldberg 1985; Munoz and Wurtz 1995). We found open visual fields and movement fields, respectively, in $18 \%(6 / 33)$ and 53\% (18/34) of SC neurons, 57\% (16/28) and $76 \%(22 / 29)$ of MD neurons, and 18\% (6/33) and 43\% (10/23) of FEF neurons. There were three basic kinds of open visual or movement fields as illustrated by representative examples in Fig. $7 D$. In some neurons, activity rose and then stayed at a plateau level for as large of eccentricities that we could test (Fig. 7D, left); these fields had no distinct peak. In other neurons, there was a distinct peak, but the firing rate then never dropped below the significance criterion level even at very large eccentricities (Fig. 7D, middle). Finally, in some neurons, the firing rate increased monotonically with eccentricity (Fig. $7 D$, right).

Visual or movement fields having distinct peaks of activity (e.g., Fig. 7, $B$ and $C$, and $D$, middle) appeared to encode a specific target location or saccadic vector, and those having firing rates that increased steadily with eccentricity (Fig. 7D, right) seemed to encode the distance of the target from the fovea (or the length of the saccade). Such visual and movement fields, carrying information about the target location or saccade vector, were found, respectively, in 100\% (33/33) and 88\% $(30 / 34)$ of the SC source neurons, 61\% (17/28) and 79\% $(23 / 29)$ of the MD relay neurons, and 100\% (33/33) and 96\% $(22 / 23)$ of the FEF recipient neurons. Signals encoding the target location or saccadic vector therefore were abundant throughout the pathway.

Quantitative comparison of the sizes and spatial locations of the visual fields (Fig. 8A) and movement fields (Fig. 8B) showed that they remained similar throughout the pathway. Looking for differences from SC to MD and from MD to FEF, we found only that the best eccentricities of visual fields in MD were slightly larger than in the $\mathrm{SC}$ or the FEF (Fig. 8A, *; $P<$ 0.025 criterion, corrected from $P<0.05$ because the MD data were used in 2 comparisons). Throughout the pathway nearly all visual and movement fields had contralateral best directions (Fig. 8, $A$ and $B$, far left).

The next step in determining if there was a projection topography was to analyze which part of the SC provided input 


\section{Measurement of Visual A and Movement Fields}

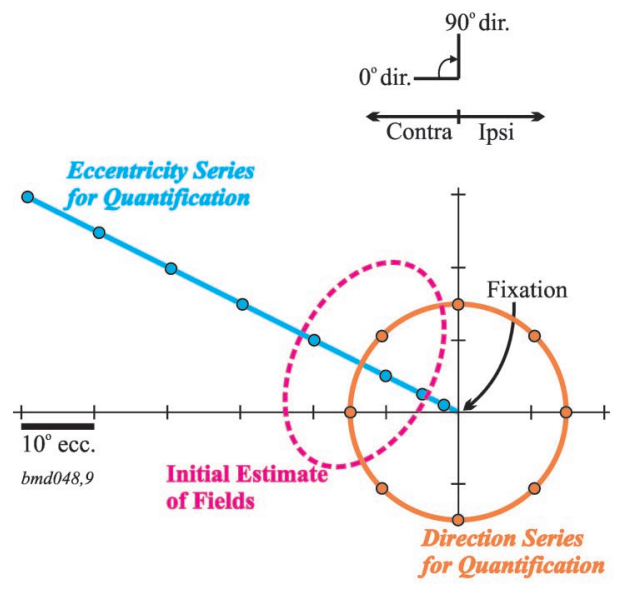

Open Movement Fields

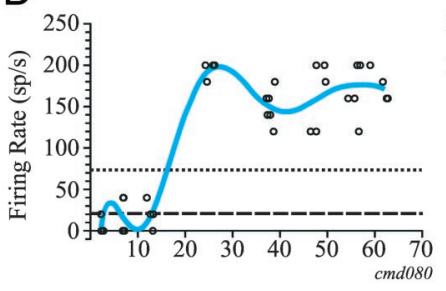

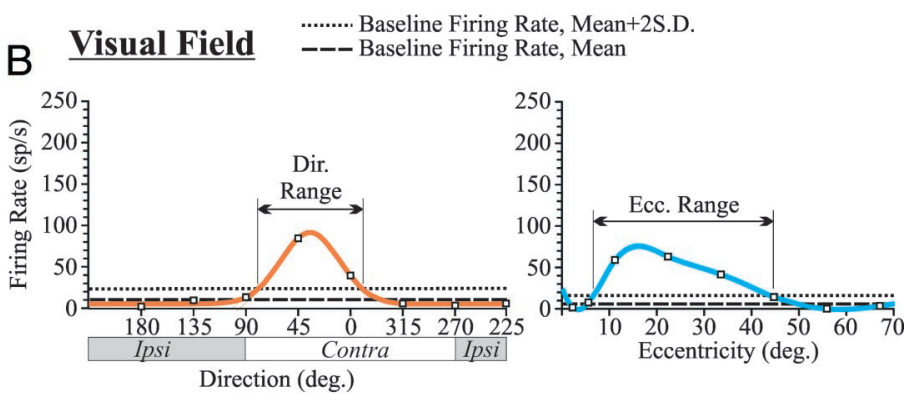

\section{Movement Field}
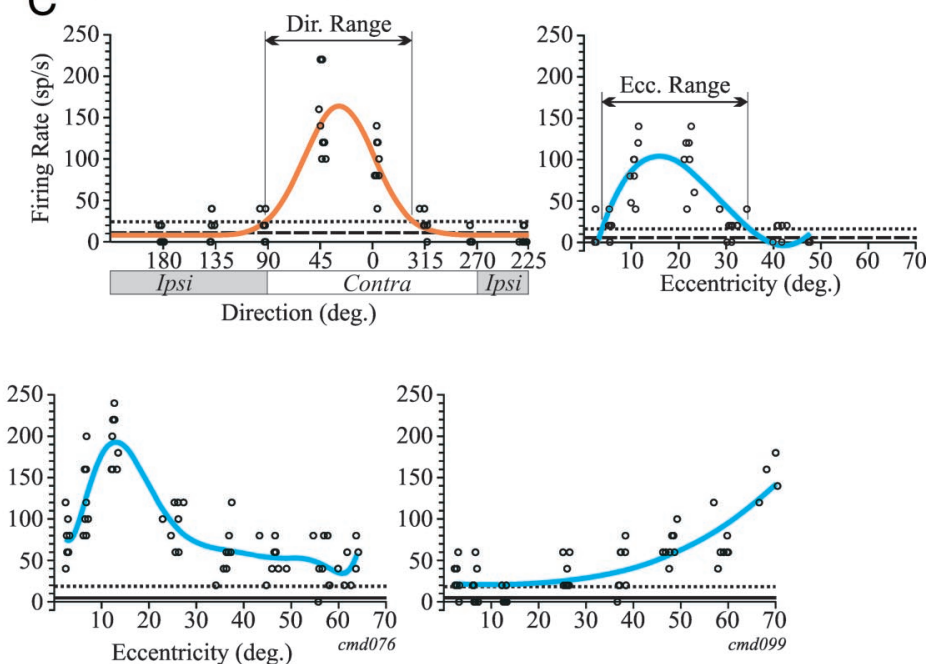

FIG. 7. Visual and movement fields. Illustrated for 1 example MD relay neuron are the method for measuring its visual and movement fields ( $A$ ), quantification of its visual field $(B)$, and quantification of its movement field $(C)$. In $B$ and $C$, direction profiles are at left and eccentricity profiles are at right. D: open movement fields of 3 other MD relay neurons.

to each MD and FEF neuron. We did this by calculating an index called the EPI (see METHODS). EPI $=-1$ meant that a neuron seemed to receive only rostral $\mathrm{SC}$ input; $\mathrm{EPI}=+1$ meant that it seemed to receive only caudal SC input; and intermediate EPI values indicated that it received input from intermediate rostrocaudal SC locations. MD and FEF neurons received input from a wide range of $\mathrm{SC}$ locations as shown by the pooled distribution of EPIs in Fig. 9A (囵; $\square$, irrelevant here, is described in DISCUSSION).

Finally, we evaluated whether there was a projection topography by plotting the best eccentricity of each MD or FEF neuron against its EPI. We used $\log _{10}$ (best eccentricity) because eccentricity is represented logarithmically across the SC, but the same result was found using nontransformed best eccentricity. $\log _{10}$ (best eccentricity) was directly correlated with EPI for both MD relay neurons (Fig. 9B, top) and FEF recipient neurons (Fig. 9B, bottom). The appreciable scatter in the correlations was probably due to noise in the measurements of best eccentricity and current threshold (used to calculate EPI). Due to time constraints, best eccentricity was found using only limited quantification of the visual and movement fields (Fig. 7A) and current threshold for activating a neuron $50 \%$ of the time was found by iterating current levels over dozens of trials rather than the hundreds of trials that would have been preferable. Regardless, the significant correlations (Fig. 9B) did indicate that MD or FEF neurons having relatively small or large eccentricity fields were preferentially activated, respec- tively, from rostral or caudal SC, consistent with an orderly projection topography.

To summarize the results, we pooled all the data (Fig. 9C) and used the resulting regression equation and the average locations of the rostral and caudal stimulating electrodes (see METHODS) to illustrate the projection topography schematically (Fig. 9D). The topography was reasonably precise: projections from $\sim 2^{\circ}$ eccentricity on the SC map preferentially drove MD or FEF neurons having visual or movement fields of $\sim 1^{\circ}$ best eccentricity, projections from $\sim 5^{\circ}$ eccentricity preferentially drove neurons having fields of $\sim 6^{\circ}$ eccentricity, and projections from $\sim 13^{\circ}$ eccentricity preferentially drove neurons having fields of $\sim 23^{\circ}$ eccentricity.

\section{Signals conveyed through the pathway}

VARIETY OF SIGNAL TYPES CARRIED BY THE NEURONS. We evaluated the neuronal signals primarily with delayed-saccade tasks (Fig. 10A). After Bruce and Goldberg (1985), we classified neurons into three major categories: visual neurons (Fig. $10 B$ ) having phasic or tonic visual activity but no presaccadic activity, visuomovement neurons (Fig. 10C) having phasic or tonic visual activity plus presaccadic activity, and movement neurons (Fig. 10D) having presaccadic activity but no phasic or tonic visual activity. We found all three categories of neurons throughout the pathway, except that there were no movement neurons in the FEF recipient neuron sample (Fig. 10D).

Figure $11 A$ shows the distributions of visual, visuomove- 
A Visual Fields

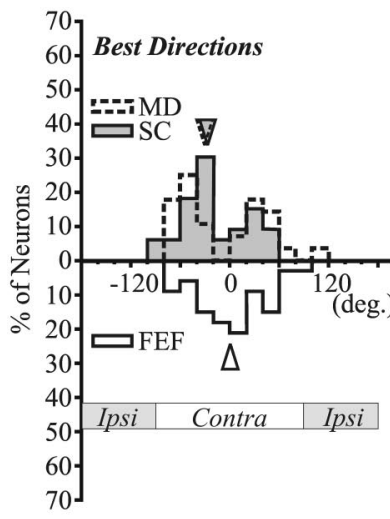

\section{$B$ Movement Fields}

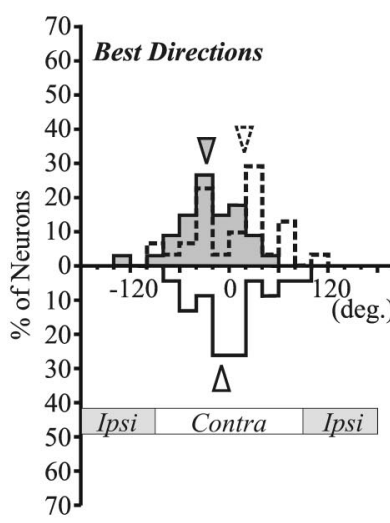

* Signif. diff. from MD ( $(i)$ distribution $(\mathbf{p}<0.025)$
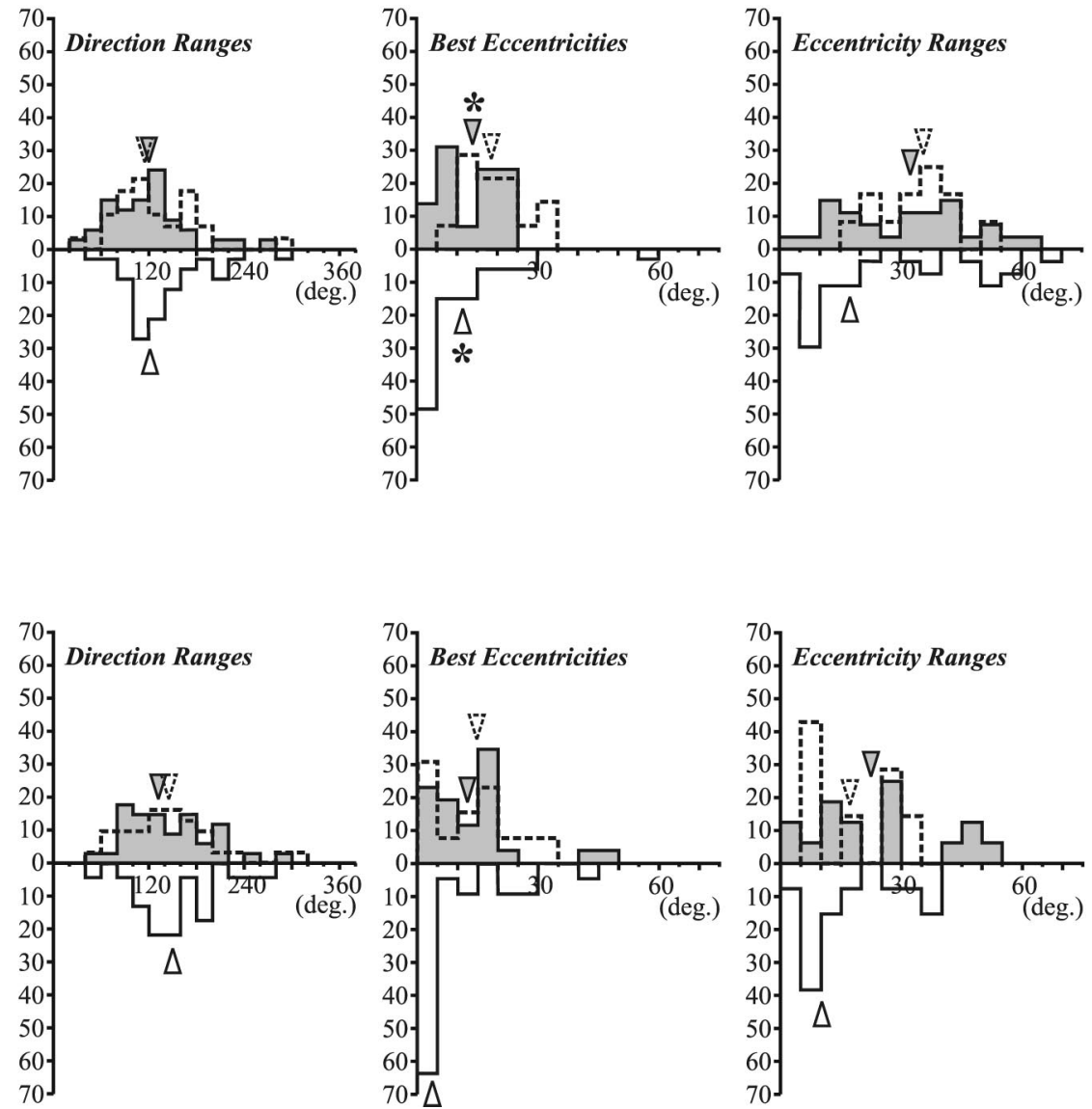

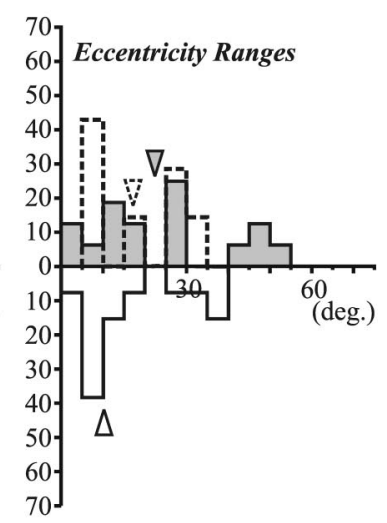

FIG. 8. Summary of best directions, direction ranges, best eccentricities, and eccentricity ranges for visual $(A)$ and movement fields $(B)$ for all 3 samples of neurons. Legend is in leftmost graph of $A$. $\triangle$, medians. The SC and FEF distributions were each compared with the MD distribution, and significant differences are indicated with asterisks. Best directions were angular data and thus required circular statistics analysis (Mardia-Watson-Wheeler test) (Batschelet 1981). For clarity, FEF distributions are inverted.

ment, and movement neurons at each stage of the pathway. Nearly every neuron in the pathway was active in the delayedsaccade tasks with only a small proportion at each stage unmodulated ("other" neurons). Visuomovement neurons were the most common type at every stage. We compared the distributions of neuron types from SC to MD and from MD to FEF ( $\chi^{2}$ tests, $P<0.025$ criterion because MD data were tested twice). From SC to MD, there was no significant change in the distribution of neuron types, but from MD to FEF, the distribution changed significantly: the FEF distribution was much more visual in nature than would be expected from its MD input.

Many of the neurons also had delay activity, a signal occurring in memory trials after target disappearance and before saccade initiation that may be involved in higher-level functions such as working memory (Fuster 1997; Goldman-Rakic 1995). As examples, delay activity was present in all of the visuomovement neurons in Fig. $10 C$ (see orange data representing memory trials). From SC to MD, the proportion of neurons with delay activity dropped significantly (Fig. 11B), but from MD to FEF, it did not change.

Because the proportions of visual, visuomovement, and movement neurons stayed the same from SC to MD (Fig. 11A), but the proportion of neurons having delay activity dropped
(Fig. 11B), the connection from SC to MD seemed to act like a high-pass filter. That is, the connection seems to let bursts of action potentials through more readily than sustained activity (visual, visuomovement, and movement neurons nearly always had sharp visual- and saccade-related bursts of activity; see Fig. 10, $B-D$ ). To test this idea, we also examined whether the proportion of neurons carrying another sustained signal, tonic visual activity, similarly decreased from SC to MD. Figure $11 C$ shows that it did. Moreover, we found that this was not a trivial result of delay activity and tonic visual activity tending to co-occur in the same neurons (analysis not shown).

Notably, however, the proportion of tonic visual signals increased from MD to FEF (Fig. 11C). Taken together with the result of Fig. 11A, that FEF recipient neurons are generally more visual than expected from their MD input, this suggests that FEF recipient neurons receive additional visual signals from elsewhere (probably from extrastriate cortex).

Many neurons in the SC and the FEF have gap activity presumably related to cognitive aspects of preparing to move or disengaging from fixation (Dias and Bruce 1994; Munoz et al. 2000), so we also used a gap task (Fig. 12A) to see if the pathway carried this signal. Gap activity is a slowly rising firing rate at the end of a gap period, occurring after a foveated spot disappears and before the neuron can possibly respond to 

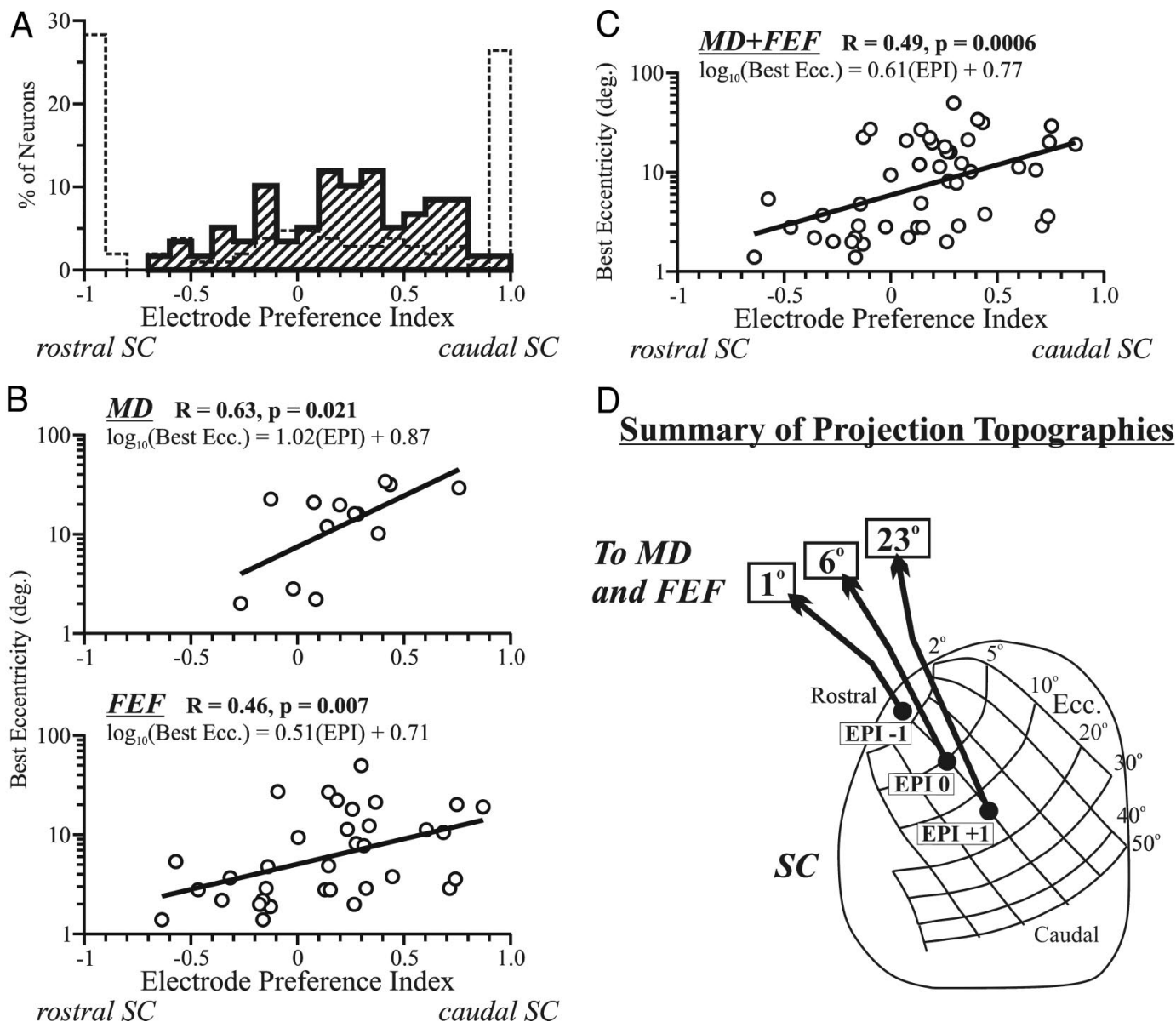

FIG. 9. Projection topographies from SC to MD and FEF. A: 四, the distribution of electrode preference indices (EPIs) for all the $\mathrm{MD}$ and FEF neurons pooled together. An EPI value indicates whether an MD or FEF neuron was activated preferentially from rostral SC (negative EPI) or caudal SC (positive EPI). For comparison (see DISCUSSION), $\square$ shows the distribution of EPIs for FEF neurons that project to the SC (from Sommer and Wurtz 2000b). Note that all neurons for which an EPI could be measured are included in these histograms, including some for which a best eccentricity did not exist or was not measured. $B$ : for MD relay neurons (top) and FEF neurons (bottom), the best eccentricity (logarithmically transformed) of the visual and/or movement field of each neuron is plotted against the EPI of the neuron. If a neuron had both a visual and a movement field, we used the average best eccentricity of the fields. $C$ : overall relationship between best eccentricity and EPI, created by pooling all the MD and FEF data from the graphs in $B$. $D$ : diagram summarizing the projection topography. This illustration was derived from the regression line equation in $C$ and the average rostral ("EPI -1") and caudal ("EPI + 1") SC electrode placements plus interpolated midrange data ("EPI 0").

the onset of the visual target for a saccade. Some neurons at every stage of the ascending pathway had gap activity (Fig. $12 B)$. The occurrence of gap activity, however, diminished significantly from SC to MD (Fig. 12C). This provides even further evidence for a high-pass filter at the SC-MD synapse, given the slowly varying nature of gap activity (albeit not as sustained as delay or tonic visual activity, it nevertheless is a much more slowly varying signal than the visual or saccadic bursts). From MD to FEF, there was no significant change in the proportions of neurons having gap activity (Fig. 12C).

Finally, we also looked for two other kinds of signals that were relatively rare: fixation-related and postsaccadic activity. A few neurons clearly changed their firing rate at the start of fixation, and to further study them, we ran monkeys on a standard fixation blink task in which the fixation spot disappeared for $\sim 400 \mathrm{~ms}$, allowing us to distinguish activity related to the motor act of fixating from activity related to foveal visual responsiveness (for details, see Sommer and Wurtz 2000b). We found $5 \mathrm{SC}$ neurons with foveal visual responses, 3 of which also had a fixation signal, 3 MD neurons with fixation signals, 2 of which had a foveal visual response, and 10 FEF neurons having the following distribution of signals: 2 had a foveal visual response but not a fixation signal, 4 had a fixation signal but not a foveal visual response, and 4 had both a foveal visual response and a fixation signal. We also searched for postsaccadic activity using the delayed-saccade tasks (see METHODS), and found three neurons in each of the SC, MD, and FEF samples that exhibited this activity.

STRENGTH AND TIMING OF SIGNALS. The preceding analyses were concerned with the percentages of various signals at different levels of the pathway; now we consider the activity profiles of the signals. We focused on visual- and saccaderelated bursts of activity because these signals were plentiful throughout the pathway. For each neuron, we constructed spike density functions to summarize its firing rate in the visual version of the delayed-saccade task. To analyze visual bursts (Fig. 13A, left), we used Gaussians of width $\sigma=2 \mathrm{~ms}$ for constructing spike density functions (narrower 
A Delayed Saccade Task

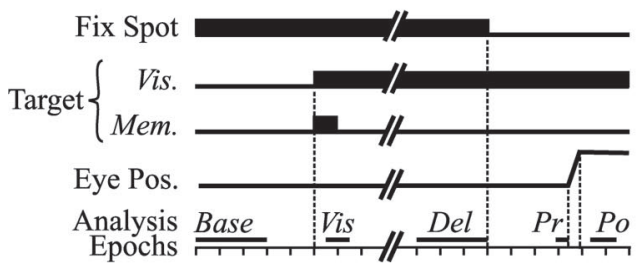

B Visual Neurons

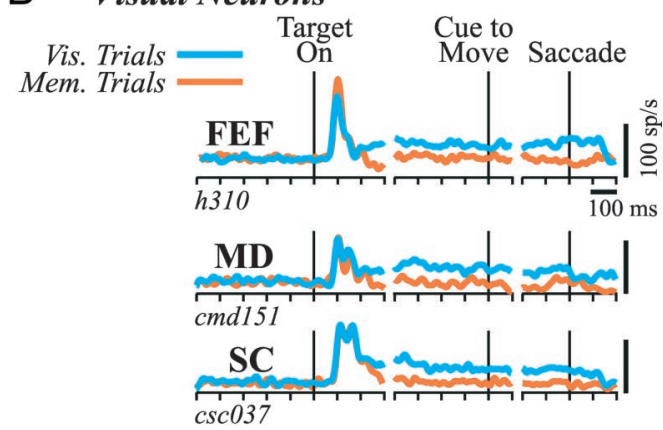

C

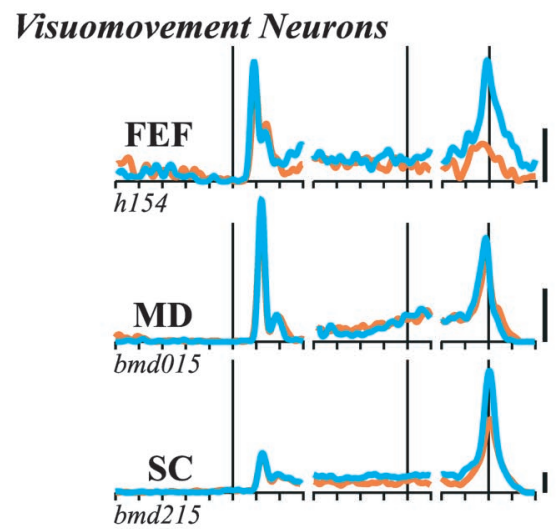

D Movement Neurons

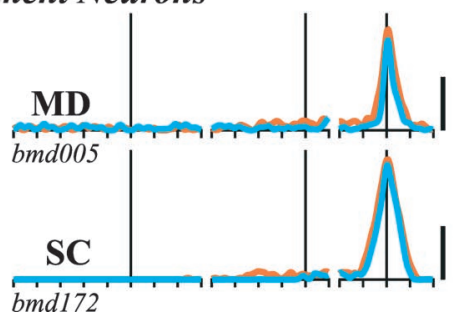

FIG. 10. Signals conveyed in the ascending pathway as determined using the delayed-saccade tasks. A: timeline of the tasks. The monkey foveated a fixation spot (Fix Spot) and then a peripheral target appeared. In the visual (Vis.) version of the task, the target remained on during an extended delay period, and in the memory (Mem.) version, it appeared for $100 \mathrm{~ms}$ and then was absent during the delay period. Disappearance of the fixation spot was the cue to make a saccade to the target location. Eye position (Eye Pos.) is shown in the fourth trace from the top. Base, baseline; Vis, visual; Del, delay; Pr, presaccadic; Po, postsaccadic. Time scale is at bottom, with ticks separated by $100 \mathrm{~ms}$. B: examples of visual neurons. Depicted are spike density functions (Gaussian width $10 \mathrm{~ms}$ ) (MacPherson and Aldridge 1979) showing the average firing rates of example FEF recipient, MD relay, and SC source neurons during the visual and memory trials. Data aligned to target onset (left), to fixation spot offset (cue to move; middle); and to saccade onset (right). Labels at lower left of each graph are identifying codes for each neuron. Further examples show visuomovement $(C)$ and movement neurons $(D)$.
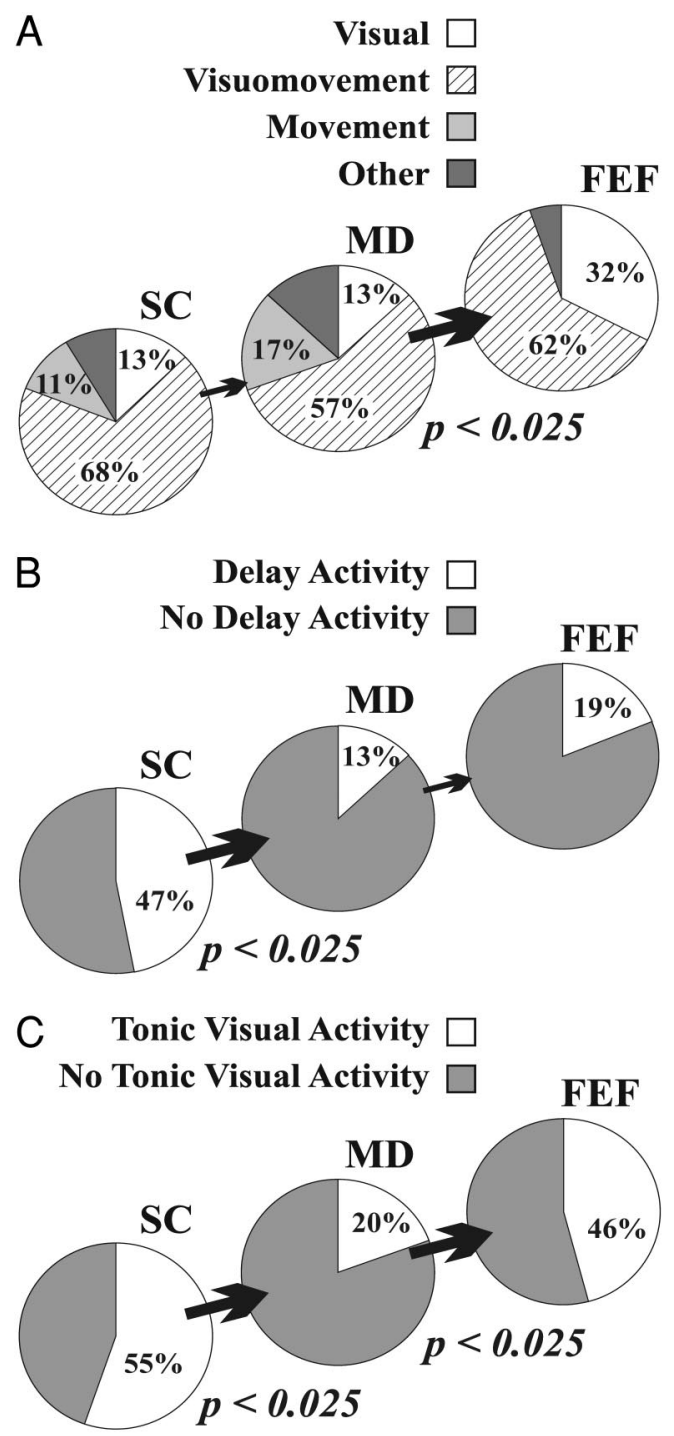

FIG. 11. Distributions of neuron classes, showing the percentages of visual, visuomovement, and movement neurons $(A)$, neurons having delay activity $(B)$, and neurons having tonic visual activity $(C)$, at each stage in the ascending pathway. Larger arrows indicate significantly different distributions. The pie charts represent $47 \mathrm{SC}$ neurons, $46 \mathrm{MD}$ neurons, and 37 FEF neurons.

Gaussians resulted in data too noisy for reliably analyzing every neuron, and wider Gaussians resulted in unacceptable underestimates of the visual latency and visual burst magnitude). The latency of a visual burst was the duration from target onset until the spike density function crossed a significance threshold set to 2 SDs above mean baseline activity (measured from $40 \mathrm{~ms}$ before to $40 \mathrm{~ms}$ after target onset). The burst magnitude was the peak magnitude of the burst minus the mean baseline activity. The peak time was the duration from target onset until the peak magnitude of the burst. In this example (Fig. 13A, left), the visual burst latency was $75 \mathrm{~ms}$, the burst magnitude was 475 spikes/s, and the peak time was $90 \mathrm{~ms}$ after target onset (also, the baseline activity had a mean of 8 spikes/s and an SD of 11 spikes/s, yielding a significance threshold of 30 spikes/s). To analyze saccadic bursts (Fig. 13A, right), we used similar methods and the same descriptors (latency, burst magnitude, and peak time) except that the spike density function Gauss- 


\section{A Gap Task}
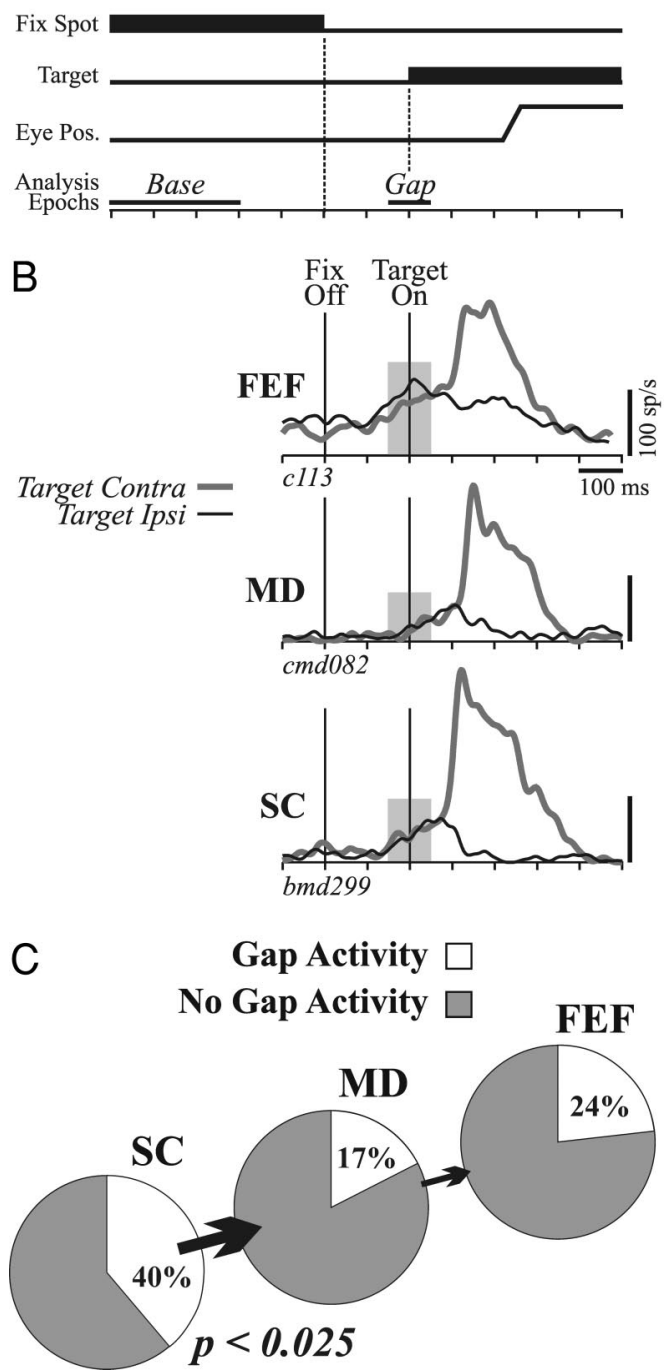

FIG. 12. Distributions of neurons having gap activity. A: timeline of the gap task. After the fixation spot was foveated, it disappeared and a gap period ensued for $200 \mathrm{~ms}$, a target appeared in the periphery, and the monkey made a saccade to the target location. Time scale is at bottom, with ticks separated by $100 \mathrm{~ms}$. $B$ : neurons with gap activity. Gap activity (shaded boxes) was an elevated firing rate from the end of the gap period to just after target onset (before any visual response of the neuron began). The gap activity occurred regardless of whether the target eventually appeared in the contra- or ipsilateral hemifield (see legend; these 2 alternatives were randomized by trial). $C$ : percentages of neurons along the pathway having gap activity. The pie charts represent $47 \mathrm{SC}, 46 \mathrm{MD}$, and $37 \mathrm{FEF}$ neurons (same neurons as in Fig. 11).

ian was set to $10 \mathrm{~ms}$ and the baseline activity was measured during the immediately preceding Delay epoch (see Fig. $10 A$ ). We tried narrower Gaussians but they often caused spurious, temporary rises above the baseline activity prior to the saccade. In this example (Fig. 13A, right), the saccadic burst latency was $-96 \mathrm{~ms}$, the burst magnitude was 194 spikes/s, and the peak time was $-8 \mathrm{~ms}$, where negative timing values represent periods before saccade initiation.

Table 1 lists all the quantitative results for the visual and saccadic bursts, and to allow a more qualitative appreciation of the data, Fig. $13 B$ shows the grand average spike density functions (created by averaging the spike density functions from each neuron). A caveat of averaging together spike density functions is that burst latencies appear shorter than they actually are; for example, FEF recipient neurons had a median visual burst latency of $84 \mathrm{~ms}$ (Table 1, top row) even though in Fig. $13 B$ (left) the latency appears to be $\sim 65 \mathrm{~ms}$. Thus although Fig. $13 B$ accurately depicts the relative differences between the SC, MD, and FEF data, one should consult Table 1 for exact timing values. The most striking result in the visual burst data were that the FEF latencies occurred earlier than the MD latencies (Fig. 13B, left; Table 1, top row). In other words, the visual response of FEF recipient neurons preceded that of the MD neurons projecting to them, and therefore the primary visual drive of the FEF recipient neurons cannot be coming from the ascending pathway. In contrast, from SC to MD, the visual burst latencies were not different (Fig. 13B, left; Table 1, top row), consistent with the SC providing the primary visual drive of the MD neurons. The peak times of visual bursts also were shorter in FEF than in MD, whereas they were not significantly different between SC and MD (Table 1, 2nd row from top). In terms of the strengths of the bursts, visual burst magnitudes were not significantly different from SC to MD or from MD to FEF (Table 1, 3rd row from top). However, the baseline activity significantly decreased from $\mathrm{SC}$ to $\mathrm{MD}$ and then significantly increased from MD to FEF (Table 1, 4th row from top). Note that these results, that the burst magnitude does not change from SC to MD but the baseline activity decreases, further support our conclusion from the previous section that the SC-MD synapse acts as a high-pass filter.

In the saccadic burst data we found no significant differences in the latency, peak time, or burst magnitude in either leg of the pathway (from SC to MD or from MD to FEF; Fig. 13B, right; Table 1, 5th-7th rows from top). The only difference was a drop in baseline activity from SC to MD and a rise from MD to FEF (Fig. 13B, right; Table 1, bottom row). This baseline was measured in the immediately preceding delay period, and because all these data come from visual trials of the delayedsaccade task, this activity essentially represents the strength of the tonic visual activity. Hence, not only did the occurrence of tonic visual activity drop from $\mathrm{SC}$ to $\mathrm{MD}$ and rise from MD to FEF (Fig. 11C), but also the strength of this activity underwent the same changes.

In the insets of Fig. 13B, the grand average spike density functions are synchronized to the start of the bursts to reveal the bursts' average shapes. The average visual bursts (Fig. 13B, left inset) were bimodal with a major peak $\sim 20 \mathrm{~ms}$ into the burst, a secondary peak $\sim 50 \mathrm{~ms}$ later, and a total duration of $\sim 150 \mathrm{~ms}$ on average. This bimodality was conserved from SC to MD to FEF. The saccadic bursts (Fig. 13B, right inset) retained approximately the same width through the pathway ( $\sim 250 \mathrm{~ms}$ ), although the average shapes of the bursts differed somewhat in SC, MD, and FEF.

In sum, these strength and timing analyses yielded three major results. First, the visual bursts of FEF recipient neurons occurred too early to result solely from ascending pathway input. These neurons must get substantial visual input from elsewhere. Second, the saccadic bursts seemed especially important as they appeared to pass unhindered through the pathway. Neither their strength nor their timing changed significantly from SC to MD to FEF. Third, all tonic activity (base- 


\section{A Single Neuron Example}

Visual Burst

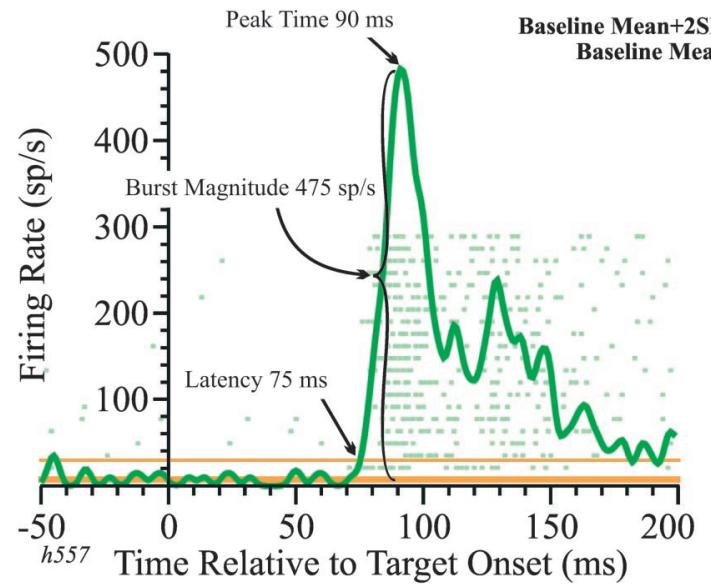

\section{B Sample Averages}
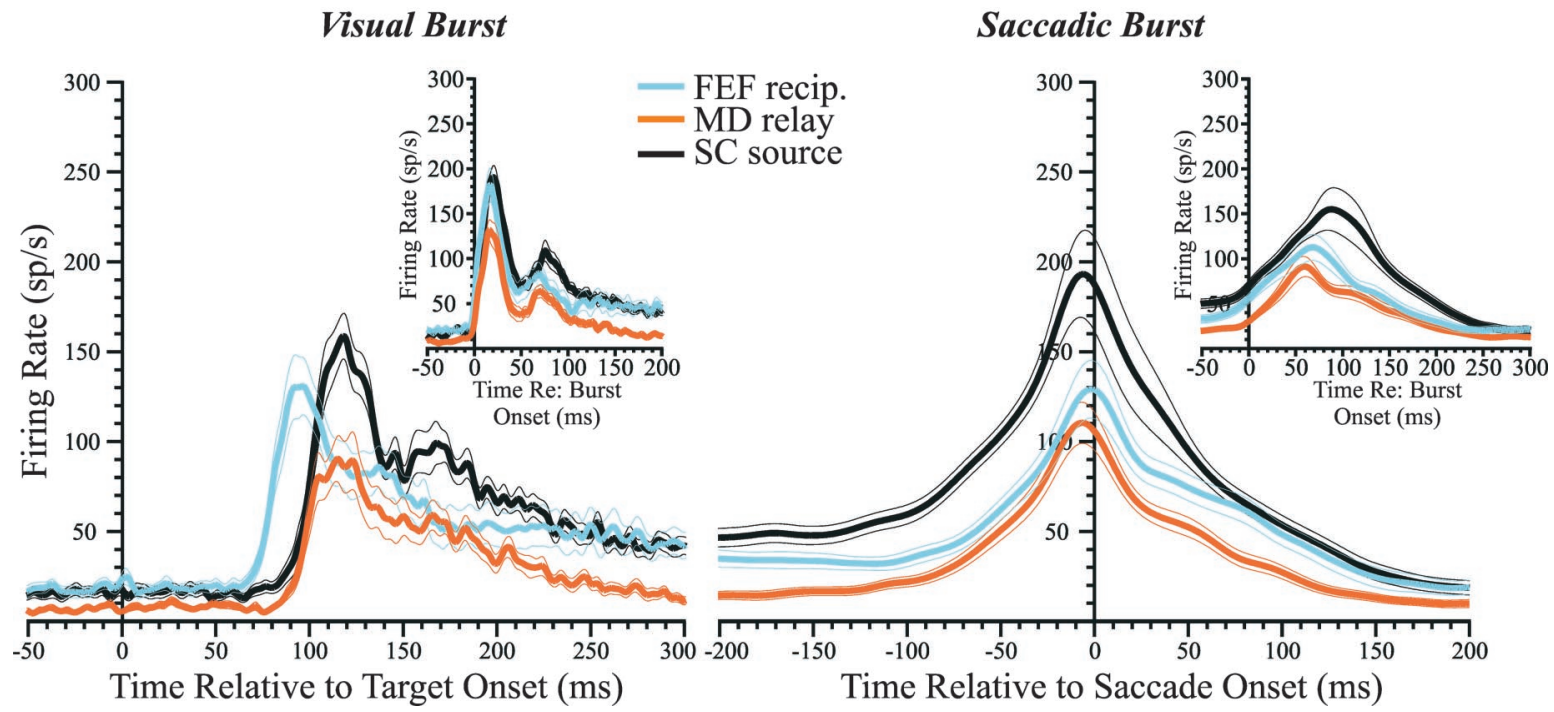

FIG. 13. Strength and timing of signals. A: single neuron data illustrating the analyses. A visual burst is analyzed at left for 1 FEF recipient neuron and a saccadic burst is analyzed at right for another. Green dots show rasters of spikes from individual trials. See RESULTS for details. $B$ : overall average visual bursts (left) and saccadic bursts $(r i g h t)$. Thick lines are means, thin lines SEs. Data were aligned to target onset (left) or saccade onset (right) in the main graphs and to start of the bursts in the insets.

line levels prior to the bursts) decreased in strength from SC to $\mathrm{MD}$, supporting the idea that this synapse acts as a high-pass filter.

GENERAL POPULATION OF SC NEURONS VERSUS THOSE PROJECTING UP TO MD. In addition to recording from identified SC source neurons, we also recorded from some unidentified single neurons $(n=17)$ and multi-unit sites $(n=23)$ in the SC intermediate layers. These neurons were "unidentified" in that we did not try activating them from any other brain structure; they are a sample of the general population of SC intermediate layer neurons. We found that the signals carried by SC source neurons were quite similar to the signals exhibited by this general sample of SC neurons as summarized in Fig. 14 and Table 2. The only differences were that SC source neurons had slightly longer latency visual responses, slightly earlier peaks in their saccadic bursts, and higher baseline firing rates. Overall there was no obvious output selectivity; the signals sent from
SC to MD were representative of those generally found within the SC intermediate layers.

\section{I S C U S S I O N}

The purpose of this study was to determine what the SC tells the FEF. We identified neurons throughout the SC-MD-FEF pathway, found that signal transmission from SC to FEF is quick and topographically organized, and concluded that multiple signals travel from SC to FEF including a salient message about the vector of the impending saccade. Figure 15 summarizes our results in terms of signal content (Fig. 15A) and timing (Fig. 15B). We next discuss these findings in detail.

\section{What the SC tells the FEF}

PREEMINENCE OF SACCADIC ACTIVITY IN THE PATHWAY. Many types of signals flow through the SC-MD-FEF pathway, but 
TABLE 1. Strength and timing of visual and saccadic signals along the pathway

\begin{tabular}{lccc}
\hline \hline & $\begin{array}{c}\text { SC Source } \\
\text { Neurons }\end{array}$ & $\begin{array}{c}\text { MD Relay } \\
\text { Neurons }\end{array}$ & $\begin{array}{c}\text { FEF Recipient } \\
\text { Neurons }\end{array}$ \\
\hline Visual burst & & & \\
$\quad$ Latency, ms & $98(11 ; 36)$ & $98(16 ; 30)$ & $84(16 ; 35)^{*}$ \\
Peak time, ms & $119(18 ; 36)$ & $119(19 ; 30)$ & $98(16 ; 35)^{*}$ \\
Burst magnitude, spikes/s & $207(93 ; 36)$ & $160(84 ; 30)$ & $150(121 ; 35)$ \\
Baseline activity, spikes/s & $12(16 ; 36)^{*}$ & $7(8 ; 30)$ & $15(14 ; 35)^{*}$ \\
Saccadic burst & & & \\
Latency, ms & $-85(39 ; 36)$ & $-66(51 ; 33)$ & $-54(38 ; 20)$ \\
Peak time, ms & $-9(13 ; 36)$ & $-3(11 ; 33)$ & $0(17 ; 20)$ \\
Burst magnitude, spikes/s & $110(93 ; 36)$ & $83(103 ; 33)$ & $89(85 ; 20)$ \\
Baseline activity, spikes/s & $36(39 ; 36)^{*}$ & $8(15 ; 33)$ & $30(28 ; 20)^{*}$ \\
\hline
\end{tabular}

Cell contents show medians (widths from 25th to 75th percentiles; $n$ ). Only neurons with significant visual bursts were included in the visual burst analyses, and only neurons with significant saccadic bursts were included in the saccadic burst analyses. * Superior colliculus (SC) or frontal eye field (FEF) distribution significantly differs from the mediodorsal thalamus (MD) distribution at $P<0.025$.

information about impending saccades seems to be particularly important. This conclusion is based on multiple lines of evidence: throughout the pathway $\sim 60-80 \%$ of the neurons have presaccadic activity (Fig. $11 \mathrm{~A}$, visuomovement + movement neurons), this activity is strong (Table 1, 7th row), it begins well before saccade initiation (Table 1, 5th row), and it peaks at or just before saccade initiation (Table 1, 6th row). Also, at every level of the pathway, the movement fields are similar (Fig. 8B), most are contraversively directed, and most carry information about the saccadic vector. Figure $15 B$ summarizes the crux of our argument. Presaccadic activity passes unimpeded from SC up to FEF and arrives just at the right time to contribute to FEF presaccadic activity, while for other signals it is a matter of "too little, too late." Too little delay activity makes it through the MD relay node for it to be a candidate as a crucial signal in the pathway, and visual signals arrive too late to be the primary cause of FEF visual responses.

What is the function of the presaccadic activity sent from SC
TABLE 2. Signals carried by SC source neurons and by the SC general population

\begin{tabular}{lcc}
\hline \hline & $\begin{array}{c}\text { SC Source } \\
\text { Neurons }\end{array}$ & $\begin{array}{c}\text { SC General } \\
\text { Population }\end{array}$ \\
\hline Visual burst & & \\
$\quad$ Latency, ms & $98(11 ; 36)^{*}$ & $92(16 ; 38)$ \\
Peak time, ms & $119(18 ; 36)$ & $115(19 ; 38)$ \\
Burst magnitude, spikes/s & $207(93 ; 36)$ & $163(93 ; 15)$ \\
Baseline activity, spikes/s & $12(16 ; 36)^{*}$ & $0(2 ; 15)$ \\
Saccadic burst & & \\
Latency, ms & $-85(39 ; 36)$ & $-72(48 ; 37)$ \\
Peak time, ms & $-9(13 ; 36)^{*}$ & $4(10 ; 37)$ \\
Burst magnitude, spikes/s & $110(93 ; 36)$ & $228(236 ; 15)$ \\
Baseline activity, spikes/s & $36(39 ; 36)^{*}$ & $11(30 ; 15)$ \\
\hline
\end{tabular}

Cell contents show medians (widths from 25th to 75th percentiles; $n$ ). Single-neuron and multi-unit data were combined to evaluate timings, but only single-neuron data were used to evaluate firing rates (multi-unit data provide accurate timing information but exaggerated firing rates). Only single neurons or multi-unit sites exhibiting significant visual bursts were included in the visual burst analyses, and only single neurons or multi-unit sites exhibiting significant saccadic bursts were included in the saccadic burst analyses. * SC source neurons significantly differ from SC general population at $P<0.025$.

to FEF? It is not proprioceptive because it starts before the eyes move. Also, it is unlikely to directly contribute to saccade generation for two reasons. First, the activity travels away from the saccade generating circuits of the pons and midbrain and instead goes up to cortex. Second, the activity does not impinge on pure movement neurons in the FEF, even though such neurons are common in the general population there (Bruce and Goldberg 1985; Schall 1991); instead, it selectively targets FEF neurons having visual responses (visual and visuomovement neurons). These three lines of evidence-that the activity is presaccadic, that it ascends into cortex, and that it influences visual-related neurons - are, however, compatible with a hypothesis that the activity is a corollary discharge. The term corollary discharge (Sperry 1950) refers to copies of movement commands used solely as information (reviewed by Bell 1984; McCloskey 1981). We hypothesize that presaccadic activity in

\section{SC Neurons: Source Neurons vs. General Population}

Visual Burst

Saccadic Burst

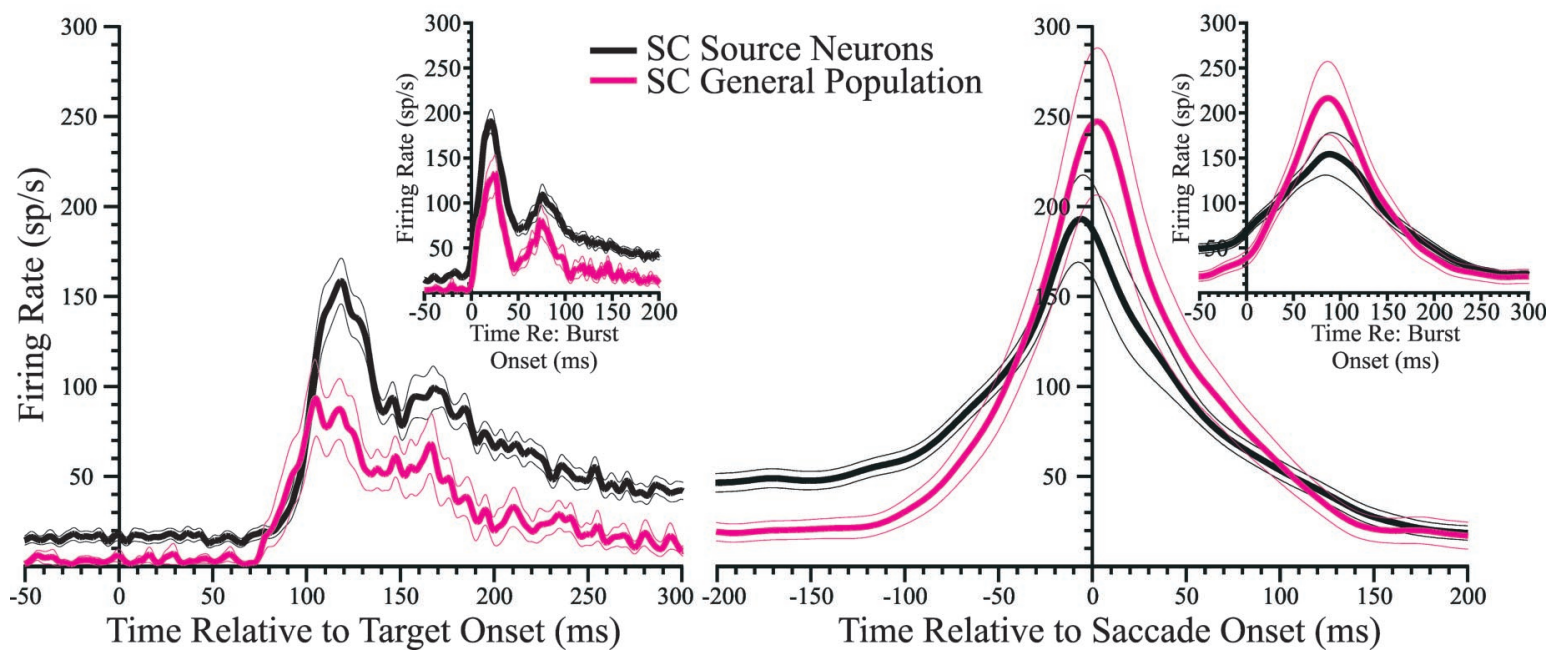

FIG. 14. Comparison of the activity ascending from the SC with the activity generally found within the SC. The overall average visual bursts (left) and saccadic bursts (right) of the identified SC source neurons and of the unidentified, general population of SC neurons were calculated and presented as in Fig. 13. 

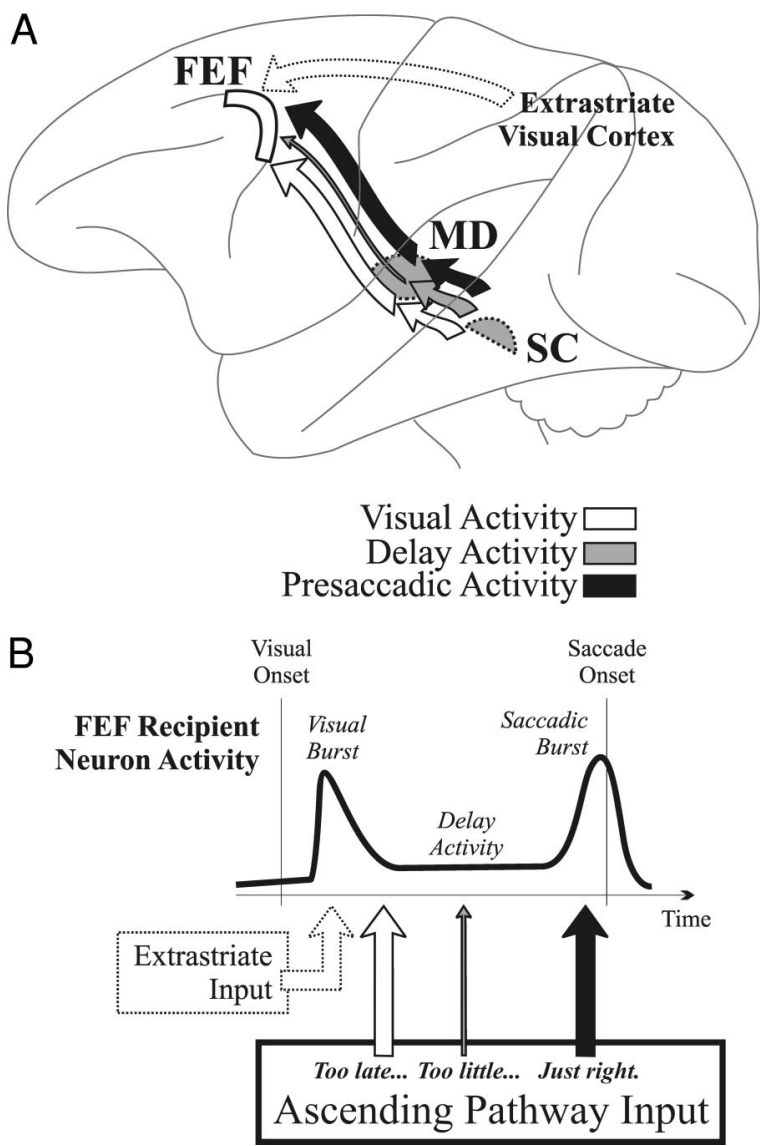

FIG. 15. Summary of what the SC tells the FEF. A: signal content in the SC-MD-FEF pathway. Visual, delay, and presaccadic activity are all sent from $\mathrm{SC}$ to $\mathrm{MD}$, and all 3 continue to FEF except that the amount of delay activity is severely reduced. At the FEF, neurons receiving this ascending input also seem to receive extra visual input, presumably from extrastriate cortex. $B$ : timing in the pathway, illustrated using a schematic activity profile of a typical FEF visuomovement cell. Visual signals from the ascending pathway arrive too late to cause the FEF visual burst, which is probably initiated by extrastriate input. It appears that too little delay activity survives through the pathway to be of major importance. However, the presaccadic activity seems just right; it travels unhindered through the pathway and arrives precisely at the appropriate time to contribute to saccadic bursts in the FEF neurons.

the SC-MD-FEF pathway acts to inform the FEF about saccadic movement commands produced by the SC. By impinging on visual-related neurons, the activity may help prepare the visual system for the abrupt changes in visual input that occur when saccades suddenly rotate the retinas (e.g., see von Helmholtz 2000). More generally, the diverse roles of the FEF not only in vision but also in motor planning, learning, and memory (reviewed by Schall 1997; Tehovnik et al. 2000) imply that it could use corollary discharge for other purposes as well, for example in coordinating saccadic sequences. In our view, therefore, an important role of the SC-MD-FEF pathway is to convey presaccadic activity that may represent corollary discharge. To test this hypothesis, we would have to interrupt signal flow in the pathway and see if this causes deficits in tasks that require corollary discharge. We have indeed done this (Sommer and Wurtz 2002), and the accompanying paper documents our findings in detail (Sommer and Wurtz 2004b).

SIGNALS THAT ASCEND FROM THE SC. We next discuss the data at a more reductionist level by stepping one at a time through each stage of the pathway. Our basic result pertaining to the first leg of the pathway was that a broad array of signals travels from SC to MD (Figs. 11 and 12). Most SC source neurons carried combinations of visual, delay, and presaccadic activity, and many had gap activity as well. All of these signals are quite common in the SC intermediate layers (Munoz et al. 2000; Sparks and Hartwich-Young 1989; Wurtz et al. 2000). Moreover, the visual and saccadic bursts of our SC source neurons were very similar to those generally found in the SC (Fig. 14). In short, the signals sent from SC to MD represent all of the signals in the SC intermediate layers, not just a subset. This is consistent with our previous hypothesis (Sommer and Wurtz 2000b) that, in the saccadic system at least, output signals of a structure closely resemble the general population of signals within that structure; selectivity is rare or absent. Lack of output selectivity also has been found for FEF (Everling and Munoz 2000; Sommer and Wurtz 2000b; see also cat results, Weyand and Gafka 1998a,b) and the lateral intraparietal area (Paré and Wurtz 1997, 2001). However, subtle differences could exist at a quantitative level between a structure's output signals and its general population of signals; for example, peak times of presaccadic bursts were slightly earlier in SC source neurons than in neurons of the general SC population (Fig. 14; Table 2, 6th row). The functional significance of such small differences is unclear.

MD RELAY NODE. Our basic finding pertaining to the relay node in the pathway was that MD neurons faithfully conveyed phasic activity (visual and presaccadic bursts) from SC to FEF but suppressed tonic activity (delay, tonic visual, and gap activity). This is suggestive of high-pass filtering, although thresholding may have contributed as well because the bursts had stronger peak firing rates than did the tonic activity. The exact mechanism is not important to the current discussion. The main point is the end result: the pathway primarily informs the FEF about when and where a visual stimulus appeared and when and where an upcoming saccade will go, information carried in the visual and saccadic bursts. In contrast, the pathway removes tonic activity and the cognitive-related signals it may convey (Fuster 1997; Goldman-Rakic 1995; Munoz et al. 2000; Wurtz et al. 2000, 2001). Leichnetz et al. (1981) hypothesized that the pathway plays a role in attention, but our data cast doubt on this considering that a prime mediator of attentional signals is tonic activity (e.g., Luck et al. 1997; Treue 2001). To the extent that attentional signals are carried in visual bursts, however, the hypothesis remains plausible.

Preferential transmission of bursts over tonic activity has been found in at least one other thalamic region, the lateral geniculate nucleus (LGN). An action potential arriving at an LGN neuron from the retina that rapidly follows another retinal input spike (within $\sim 20 \mathrm{~ms}$ ) is particularly effective in evoking an action potential from the neuron (Levine and Cleland 2001; Mastronarde 1987; Rowe and Fischer 2001; Usrey et al. 1998). Also, all thalamic relay neurons alternate between "tonic mode," in which they reproduce their input fairly linearly with gain $\leq 1$, and "burst mode," in which a single input spike can elicit a burst of spikes. In behaving monkeys, LGN neurons are usually in tonic mode but can slip into burst mode (Ramcharan et al. 2000a,b). MD relay neurons may be normally in low-gain tonic mode but occasionally shift into burst mode as the monkey anticipates target onset or saccade initiation. A sudden 
volley of spikes from the SC could then trigger an unusually vigorous burst from the MD neurons.

Guillery and Sherman (2002) suggested on the basis of our brief report (Sommer and Wurtz 2002) that our MD relay neurons might receive a modulatory input, rather than a driving input, from the SC. This seems unlikely in light of the present results. We showed here that single pulse stimulation in the SC activated MD neurons very quickly (median: $1.4 \mathrm{~ms}$, including a presumed synaptic delay of only $0.57 \mathrm{~ms}$ ) and required normal current thresholds for this type of study (mean: 264 $\mu \mathrm{A})$. The SC-MD synapse therefore was fast and strong, more like a driver than a modulator. Furthermore the firing characteristics of SC source neurons and MD relay neurons were quite similar, consistent with the former driving the latter (Figs. $11 A$ and $13 B$ ). Guillery and Sherman (2002) proposed that more likely candidates as drivers of the MD relay neurons might be descending inputs from cortex. However, the rat anatomy paper they cited as evidence for a modulatory SC input onto MD neurons stated that "collicular boutons tend to be larger and distribute to more proximal parts of the dendrites than those from the prefrontal cortex." (Kuroda and Price 1991; p. 550). Thus SC inputs should have more of an influence on MD thalamus than cortical inputs. Also, the SC inputs made asymmetric, excitatory synapses onto the thalamic neurons, and we see no reason to assume they cannot drive them. We think the evidence clearly favors a driving, not a modulatory, role for SC inputs onto MD neurons in this particular circuit.

FEF RECIPIENT NEURONS. Our basic findings regarding the FEF recipient neurons concerned the prevalence and timing of their visual responses. First, visual activity was nearly ubiquitous in the neurons. This had implications for a corollary discharge function of the SC-MD-FEF pathway, as discussed in the preceding text, and also suggested that the FEF recipient neurons get additional visual signals from elsewhere, since their input from MD was significantly less visual (Fig. 11A). Second, visual responses of the FEF recipient neurons started before those of MD relay neurons (Fig. 13B left). This further supported the argument that FEF recipient neurons receive extra visual input, and a reasonable guess is that this comes from extrastriate cortex.

Although the visual signals sent from MD to FEF were relatively slow, they were nevertheless abundant and strong. A possible function for these visual inputs might be to modulate visual signals arriving from extrastriate cortex. For example, the ascending inputs might contribute to changes in the initial visual response related to saccadic target selection ("saccadic enhancement" effects: Goldberg and Bushnell 1981; Wurtz and Mohler 1976; "target discrimination" effects: Schall and Thompson 1999). This possibility is supported by our finding that most of the visually responsive SC source neurons and MD relay neurons also had presaccadic activity (visuomovement neurons). The visual activity could be a sort of preparatory corollary discharge signal, telling the FEF which stimulus will be targeted by the upcoming saccade, prior to the presaccadic activity that represents the actual corollary discharge of the saccade. The visual activity thus should be modulated in Go/ Nogo tasks, being larger in Go trials when a stimulus will be targeted by a saccade than in Nogo trials when the stimulus will be irrelevant. Further experiments are needed to test this prediction.

Alternatively, of course, visual signals sent upstream by SC and MD neurons might simply be pure visual responses and not modulated by behavioral context. Two papers previously hypothesized that much of the visual activity in the prefrontal cortex, including in the FEF, might come from SC (Mohler et al. 1973; Suzuki and Azuma 1983). This was because FEF visual receptive fields are relatively large and unselective for visual attributes like line orientation, making them more reminiscent of SC visual receptive fields than extrastriate cortical visual receptive fields. With regard to the early phase of the FEF visual response, this hypothesis is not supported by our data because short-latency FEF visual responses cannot come from the SC. It is possible that the later phase of the FEF visual response, however, is driven partly by $\mathrm{SC}$ input. If so, a testable prediction is that the initial visual response in FEF may exhibit marked visual tuning (e.g., for orientation) and relatively small receptive fields, reflecting input solely from extrastriate cortex, but later in the response the visual tuning might broaden and the receptive fields expand, reflecting additional input arriving from the SC.

Because of the relatively late arrival of visual information from the ascending pathway into FEF, one might expect the FEF visual response to be bimodal, with an early "extrastriate input" burst followed $21 \mathrm{~ms}$ later (see Table 1, 2nd row) by a secondary "ascending pathway input" burst. Whether this occurred is difficult to say. On the one hand, the average shape of all the visual bursts in this study was bimodal (Figs. $13 B$ and 14, left insets), but visual bursts in FEF were not any more bimodal than those in $\mathrm{SC}$ and $\mathrm{MD}$, and the time lag between the peaks, $\sim 50 \mathrm{~ms}$, was longer than the expected $21 \mathrm{~ms}$. However, that all the visual responses were bimodal is not easy to explain in itself. It may be that bimodality starts in the FEF recipient neurons because of the two out-of-phase visual inputs and then propagates down to SC through corticotectal projections, inducing bimodality everywhere including in the ascending pathway back to FEF. Modeling this circuit might shed light onto why in the steady state condition the separation between peaks becomes, on average, $\sim 50 \mathrm{~ms}$ apart everywhere.

\section{Issues of methodology}

IMPORTANCE OF PHYSIOLOGICAL IDENTIFICATION. One might argue that our antidromic and orthodromic identification methods were unnecessary-perhaps the SC-MD-FEF pathway could have been adequately described by just comparing the general populations of SC, MD, and FEF neurons with each other. Neurons in each of these structures, however, are interconnected with wide-ranging parts of the brain and may be part of numerous possible networks. Only through physiological identification could we be confident that a neuron belonged to the SC-MD-FEF pathway. The activity of our identified neurons was sometimes quite different from that reported previously for general populations of neurons. For example, as noted in the preceding text, our FEF recipient neurons seemed more visual in nature than neurons in the general FEF population. Also, our MD relay neurons rarely had delay activity, which seemed surprising considering the many prior reports of delay activity in neurons of MD and nearby thalamic regions 
(Fuster and Alexander 1971; Tanibuchi and Goldman-Rakic 2003; Watanabe et al. 2000; Wyder et al. 2003). Results such as these had important implications as to the function of the pathway and were critically dependent on physiologically identifying the neurons.

POSSIBLE ORTHODROMIC ACTIVATION THROUGH COLLATERALS There is always an element of ambiguity about orthodromic activation because it can occur through routes other than the assumed one. We think that when we stimulated the SC and orthodromically drove MD and FEF neurons, this activation occurred via the ascending pathway. It could be, however, that it occurred via collaterals of corticotectal neurons ("collateral hypothesis"). Many cortical neurons project to the SC, and SC stimulation can antidromically activate them. An antidromically evoked action potential traveling in the axon of such a cortical neuron could invade a collateral terminating on an MD or FEF neuron. If input from this collateral causes the postsynaptic neuron to fire, one might mistakenly think that the neuron was being orthodromically driven through the ascending pathway.

We emphasize first that even if one completely ignores our orthodromic activation results because of this caveat, the basic conclusion of this paper remains the same. From antidromic activation results alone we know that intermediate layer SC neurons send presaccadic activity and other signals to the lateral edge of MD and that neurons there send similar signals, except with tonic activity diminished, into the FEF. These results still indicate that the pathway is highly active during visuosaccadic behavior and that presaccadic activity is one of the main signals carried through it.

In our opinion, however, the orthodromic activation results are valid; we are confident for several reasons that in our experiments the collateral hypothesis is wrong. The first reason is based on our stimulation-related data. If the collateral hypothesis were correct, stimulation in the SC should always directly activate the same neuronal elements (axons at or near their termination) regardless of whether the later event at the recorded neuron were antidromic invasion of the soma or orthodromic activation via synapses. Our EPI data, however, indicate that the same neuronal elements in the SC are not directly activated in the two situations. Recall that the EPI is an index summarizing how easy it is to drive a neuron from rostral versus caudal SC. We previously described the EPI distribution for antidromically activating FEF neurons from the SC (Sommer and Wurtz 2000b), and this distribution was strikingly bimodal (Fig. 9A, $\square$ ); more than half of the FEF corticotectal neurons could be activated only from the rostral electrode $(\mathrm{EPI}=-1)$ or only from the caudal electrode $(\mathrm{EPI}=1)$. When orthodromically activating FEF neurons from the SC in the present study, however, the EPI distribution was broadly unimodal; nearly every neuron could be driven from both the rostral and caudal SC electrode (Fig. 9A, 四). The vastly different EPI distributions strongly suggest that different neuronal elements in the SC are recruited in antidromic versus orthodromic activation-probably axon terminals during antidromic activation but axon hillocks and initial segments during orthodromic activation-and this seems to rule out the collateral hypothesis.

A second reason to doubt the collateral hypothesis pertains to activation latencies. The latencies of activating FEF recipi- ent neurons from the SC were quite precisely predicted by the latencies of signals passing through MD relay neurons (Fig. $6 B)$. The simplest explanation for this is that all activations passed through the ascending pathway from SC to MD to FEF.

A third reason to doubt the collateral hypothesis is based on studies of other pathways. Investigators stimulating the LGN to orthodromically activate striate cortex (V1) neurons, for example, have been concerned about inadvertently activating the neurons via collaterals of corticothalamic neurons. They were in the fortunate position, however, of being able to directly test this by stimulating the optic tract and seeing if the V1 neurons still fired; this could occur only if LGN efferents drove the V1 neurons. When this test has been performed, nearly every V1 neuron activated orthodromically at short latency from LGN also has been activated from the optic tract (e.g., Bullier and Henry 1979; Ferster and Lindström 1983; Singer et al. 1975; Stone and Dreher 1973; Toyama et al. 1974). One can drive V1 neurons via collaterals, but only by increasing the stimulation intensity 5- to 10-fold (Ferster and Lindström 1983, 1985). Similarly, in neurons of primary motor cortex (M1), excitatory postsynaptic potentials arising from thalamic input are more potent than those arising from collaterals of pyramidal tract neurons; membrane potentials from thalamic input are shorterlatency, faster-rising, and much more likely to evoke action potentials (Deschênes et al. 1979). In sum, collaterals in V1 and M1 are relatively weak and we see no reason why they would be stronger elsewhere.

There are also further reasons to doubt the collateral hypothesis as we discussed previously (Sommer and Wurtz 1998). These multiple lines of evidence deem it unlikely that spurious orthodromic activation via collaterals substantially affected our data.

POSSIBLE TERMINATION OF SC SOURCE NEURONS ONTO NONRELAY NEURONS. Our SC source neurons projected to lateral MD, as shown by antidromic activation, but some may have terminated on thalamic neurons that do not project to the FEF (Fig. 2B). We doubt that this confound was a major factor because we were careful to activate SC neurons only from sites of MD relay neurons and because the highly similar activity patterns of SC source neurons and MD relay neurons were consistent with their being connected.

COMPARING SIGNAL CONTENT BETWEEN AREAS. An analytical limitation pertains to our comparison of signal types at each stage of the ascending pathway. We felt we could infer how signals change from area to area because we studied only neurons known to be in the pathway. This seems better than the usual practice of comparing populations of neurons recorded with no regard to their connections (e.g., Alexander and Crutcher 1990a,b; Crutcher and Alexander 1990). However, even improved inferences are still inferences. Some might be incorrect. For example, the presaccadic bursts of FEF recipient neurons were similar in every way to the presaccadic bursts of MD relay neurons, so a logical inference is that the FEF bursts were derived from MD input. It is possible, however, that much or all of the FEF presaccadic activity came from elsewhere. Presaccadic input from MD might only create postsynaptic potentials in FEF neurons that do not cause action potentials but modify other inputs. Our corollary discharge hypothesis makes no strong prediction about this. Although SC neurons must drive MD neurons so that presaccadic activity is 
relayed accurately, the influence of MD input on FEF neurons could be multifold: MD input could modulate FEF neurons to immediately influence saccadic planning or visual analysis, it could drive them so as to relay corollary discharge onward to other neurons or networks that use the information, or it could have both influences, modulating some FEF neurons and driving others.

Despite the limitations involved in comparing signal types across areas, we still think this type of analysis has great merit in describing signal flow in a pathway as long as all neurons under consideration are identified as belonging to the pathway. At the very least it provides a first-order description that may be refined by later experiments, such as cross-correlation studies to see if neurons dually recorded in two connected areas have the predicted relationship, or reversible inactivation studies to see if inhibiting input from one area (e.g., MD) predictably changes the activity of neurons in another area (e.g., FEF).

\section{Other ascending pathways}

Many other ascending pathways remain to be explored in the awake, behaving monkey. For example, the FEF is the target of disynaptic pathways originating not only from the SC but also from the substantia nigra pars reticulata, relayed via ventroanterior thalamus, and from the dentate nucleus of the cerebellum, relayed via ventrolateral thalamus (Lynch et al. 1994; Middleton and Strick 2000). Another pathway, from superficial SC to pulvinar to extrastriate cortex, has been examined by looking at the general populations of neurons at all three levels (reviewed by Sommer and Wurtz in 2004a), but so far only one study has investigated an identified class of neurons in the pathway (superficial SC source neurons: Marrocco et al. 1981). Other ascending pathways are implied by signals found in cortex that must originate subcortically (e.g., vestibular-related activity) (Andersen 1997; Brandt and Dieterich 1999), by anatomical demonstrations of myriad brain stem inputs to thalamus (Steriade et al. 1997), and by results of psychophysical studies (e.g., Tanaka 2003). In the skeletomotor system, prominent pathways course from the deep cerebellar nuclei and pallidum through thalamus to motor cortex (for reviews see Middleton and Strick 2000; Sommer 2003). They have been studied with identified neuron methods extensively in anesthetized, paralyzed cats (e.g., Cohen et al. 1962; Deschênes and Hammond 1980; Futami et al. 1986; Henneman et al. 1950; Shinoda et al. 1985a,b) but only rarely in behaving monkeys (e.g., Anderson and Turner 1991; Holdefer et al. 2000; Nambu et al. 1991).

We suggest that the overall approach taken in studying the SC-MD-FEF pathway might serve as a good model for studying these other pathways. This approach was as follows: the anatomy of the pathway was thoroughly established (e.g., Lynch et al. 1994), neurons at all levels of the pathway were identified using ortho- and antidromic stimulation methods, and the signals encoded by the neurons were deciphered by having monkeys perform a variety of tasks. These methods, and complementary ones such as simultaneous recordings in multiple areas, hold great promise for eventually describing ascending pathways at a level of detail that may someday approach our understanding of the more traditionally studied routes coursing across cerebral cortex and down to spinal cord and brain stem.

\section{Conclusion}

Schlag-Rey and Schlag (1989) ended a comprehensive review of oculomotor thalamus by writing, "at the present time we know some of the signals and some of the lines [in thalamus], but not the signal flow through the lines. The story of the central thalamus is still very incomplete." In this study, we took initial steps toward completing the story. We discovered that a variety of signals are sent from SC to MD to FEF, with presaccadic signals appearing to be the most important. We now have a good idea about what the SC tells the FEF, but a major question remains: what is the purpose of this ascending presaccadic activity? A strong possibility discussed in the preceding text is that it plays a corollary discharge function, informing the cerebral cortex about upcoming saccades, and this hypothesis is tested in the accompanying paper (Sommer and Wurtz 2004b).

\section{A C K N OW LEDGMENTS}

We thank our colleagues in the Laboratory of Sensorimotor Research and Prakash Kara for their helpful comments, Mitchell K. Smith for his histological expertise, and the Laboratory of Diagnostic Radiology Research at the National Institutes of Health for MRI.

\section{REFEREN C ES}

Aizawa $\mathbf{H}$ and Wurtz RH. Reversible inactivation of monkey superior colliculus. I. Curvature of saccadic trajectory. J Neurophysiol 79: 2082-2096, 1998

Alexander GE and Crutcher MD. Preparation for movement: neural representations of intended direction in three motor areas of the monkey. $\mathrm{J} \mathrm{Neu}$ rophysiol 64: 133-150, 1990a.

Alexander GE and Crutcher MD. Neural representations of the target (goal) of visually guided arm movements in three motor areas of the monkey. J Neurophysiol 64: 164-178, $1990 \mathrm{~b}$.

Andersen RA. Multimodal integration for the representation of space in the posterior parietal cortex. Philos Trans R Soc Lond B Biol Sci 352: 1421$1428,1997$.

Anderson ME and Turner RS. Activity of neurons in cerebellar-receiving and pallidal-receiving areas of the thalamus of the behaving monkey. J Neurophysiol 66: 879-893, 1991.

Barbas $\mathbf{H}$ and Mesulam M-M. Organization of afferent input to subdivisions of area 8 in the rhesus monkey. J Comp Neurol 200: 407-431, 1981.

Batschelet E. Circular Statistics in Biology. New York: Academic, 1981, p. 101-104.

Bell CC. Effects of motor commands on sensory inflow, with examples from electric fish. In: Comparative Physiology: Sensory Systems, edited by Bolis L and Keynes RD. Cambridge, UK: Cambridge Univ. Press, 1984.

Benevento LA and Fallon JH. The ascending projections of the superior colliculus in the rhesus monkey (Macaca mulatta). J Comp Neurol 160: 339-362, 1975.

Bizzi E. Discharge of frontal eye field neurons during saccadic and following eye movements in unanesthetized monkeys. Exp Brain Res 6: 69-80, 1968.

Brandt $\mathbf{T}$ and Dieterich $M$. The vestibular cortex. Its locations, functions, and disorders. Ann NY Acad Sci 871: 293-312, 1999.

Bruce CJ and Goldberg ME. Primate frontal eye field. I. Single neurons discharging before saccades. J Neurophysiol 53: 603-635, 1985.

Bullier $\mathbf{J}$ and Henry GH. Ordinal position of neurons in cat striate cortex. J Neurophysiol 42: 1251-1263, 1979.

Cohen B, Housepian EM, and Purpura DP. Intrathalamic regulation of activity in a cerebellocortical projection pathway. Exp Neurol 6: 492-506, 1962.

Crist CF, Yamasaki DSG, Komatsu H, and Wurtz RH. A grid system and a microsyringe for single cell recording. J Neurosci Methods 26: 117-122, 1988

Crutcher MD and Alexander GE. Movement-related neuronal activity selectively coding either direction or muscle pattern in three motor areas of the monkey. J Neurophysiol 64: 151-163, 1990.

Deschênes M and Hammond C. Physiological and morphological identification of ventrolateral fibers relaying cerebellar information to the cat motor cortex. Neuroscience 5: 1137-1141, 1980. 
Deschênes M, Labelle A, and Landry P. A comparative study of ventrolateral and recurrent excitatory postsynaptic potentials in large pyramidal tract cells in the cat. Brain Res 160: 37-46, 1979.

Deschênes M, Landry P, and Clercq M. A reanalysis of the ventrolateral input in slow and fast pyramidal tract neurons of the cat motor cortex. Neuroscience 7: 2149-2157, 1982.

Dias EC and Bruce CJ. Physiological correlate of fixation disengagement in the primate's frontal eye field. J Neurophysiol 72: 2532-2537, 1994.

Evarts EV. Relation of pyramidal tract activity to force exerted during voluntary movement. J Neurophysiol 31: 14-27, 1968.

Everling S and Munoz DP. Neuronal correlates for preparatory set associated with pro-saccades and anti-saccades in the primate frontal eye field. $\mathrm{J} \mathrm{Neu}$ rosci 20: 387-400, 2000

Ferster D and Lindström S. An intracellular analysis of geniculo-cortical connectivity in area 17 of the cat. J Physiol 342: 181-215, 1983.

Ferster D and Lindström S. Augmenting responses evoked in area 17 of the cat by intracortical axon collaterals of cortico-geniculate cells. J Physiol 367: 217-232, 1985.

Fries W. Cortical projections to the superior colliculus in the macaque monkey: a retrograde study using horseradish peroxidase. J Comp Neurol 230: 55-76, 1984.

Fuster JM. The Prefrontal Cortex. Anatomy, Physiology, and Neuropsychology of the Frontal Lobe. Philadelphia, PA: Lippincott-Raven, 1997, p. $125-134$

Fuster JM and Alexander GE. Neuron activity related to short-term memory. Science 173: 652-654, 1971 .

Futami T, Kano M, Sento S, and Shinoda Y. Synaptic organization of the cerebello-thalamo-cerebral pathway in the cat. III. Cerebellar input to corticofugal neurons destined for different subcortical nuclei in areas 4 and 6 . Neurosci Res 3: 321-344, 1986.

Gallyas F. Silver staining of myelin by means of physical development. Neurol Res 1: 203-209, 1979.

Giguere M and Goldman-Rakic PS. Mediodorsal nucleus: areal, laminar, and tangential distribution of afferents and efferents in the frontal lobe of rhesus monkeys. J Comp Neurol 277: 195-213, 1988.

Goldberg ME and Bushnell MC. Behavioral enhancement of visual responses in monkey cerebral cortex. II. Modulation in frontal eye fields specifically related to saccades. J Neurophysiol 46: 773-787, 1981.

Goldman-Rakic PS. Cellular basis of working memory. Neuron 14: 477-485, 1995.

Goldman-Rakic PS and Porrino LJ. The primate mediodorsal (MD) nucleus and its projection to the frontal lobe. J Comp Neurol 242: 535-560, 1985

Guillery RW and Sherman SM. The thalamus as a monitor of motor outputs. Philos Trans R Soc Lond B Biol Sci 357: 1809-1821, 2002.

Harting JK, Huerta MF, Frankfurter AJ, Strominger NL, and Royce GJ. Ascending pathways from the monkey superior colliculus: an autoradiographic analysis. J Comp Neurol 192: 853-882, 1980.

Henneman E, Cooke PM, and Snider RS. Cerebellar projections to the cerebral cortex. Res Pub Assoc Res Nerv Ment Dis 30: 317-333, 1950.

Holdefer RN, Miller LE, Chen LL, and Houk JC. Functional connectivity between cerebellum and primary motor cortex in the awake monkey. $\mathrm{J} \mathrm{Neu}$ rophysiol 84: 585-590, 2000.

Kievit J and Kuypers HGJM. Subcortical afferents to the frontal lobe in the rhesus monkey studied by means of retrograde horseradish peroxidase transport. Brain Res 85: 261-266, 1975.

Kuroda M and Price JL. Ultrastructure and synaptic organization of axon terminals from brain stem structures to the mediodorsal thalamic nucleus of the rat. J Comp Neurol 313: 539-552, 1991.

Le Gros Clark WE, and Boggon RH. VII-the thalamic connections of the parietal and frontal lobes of the brain in the monkey. Philos Trans $R$ Soc Lond B Biol Sci 224: 313-359, 1935.

Leichnetz GR, Spencer RF, Hardy SGP, and Astruc J. The prefrontal corticotectal projection in the monkey: an anterograde and retrograde horseradish peroxidase study. Neuroscience 6: 1023-1041, 1981

Lemon R. Methods for neuronal recording in conscious animals. IBRO Handbook Series: Methods in the Neurosciences. New York: Wiley, 1984, vol. 4, p. 95-102.

Levine MW and Cleland BG. An analysis of the effect of retinal ganglion cell impulses upon the firing probability of neurons in the dorsal lateral geniculate nucleus of the cat. Brain Res 902: 244-254, 2001.

Lipski J. Antidromic activation of neurones as an analytic tool in the study of the central nervous system. J Neurosci Methods 4: 1-32, 1981.
Luck SJ, Chelazzi L, Hillyard SA, and Desimone R. Neural mechanisms of spatial selective attention in areas V1, V2, and V4 of macaque visual cortex. J Neurophysiol 77: 24-42, 1997.

Lynch JC, Hoover JE, and Strick PL. Input to the primate frontal eye field from the substantia nigra, superior colliculus, and dentate nucleus demonstrated by transneuronal transport. Exp Brain Res 100: 181-186, 1994.

MacPherson JM and Aldridge JW. A quantitative method of computer analysis of spike train data collected from behaving animals. Brain Res 175: 183-187, 1979.

Marrocco RT, McClurkin JW, and Young RA. Spatial properties of superior colliculus cells projecting to the inferior pulvinar and parabigeminal nucleus of the monkey. Brain Res 222: 150-154, 1981.

Martin RF and Bowden DM. Template Atlas of the Primate Brain. Seattle, WA: University of Washington Primate Information Center, 1997, p. 45-49.

Mastronarde DN. Two classes of single-input X-cells in cat lateral geniculate nucleus. II. Retinal inputs and the generation of receptive-field properties. J Neurophysiol 57: 381-413, 1987.

McCloskey DI. Corollary discharges: motor commands and perception. In: Handbook of Physiology. The Nervous System. Motor Control, edited by Brooks VB. Bethesda, MD: Am. Physiol. Soc., 1981, sect. 1, vol. II, p. 1415-1447.

Middleton FA and Strick PL. Basal ganglia and cerebellar loops: motor and cognitive circuits. Brain Res Brain Res Rev 31: 236-250, 2000.

Mohler CW, Goldberg ME, and Wurtz RH. Visual receptive fields of frontal eye field neurons. Brain Res 61: 385-389, 1973.

Munoz DP, Dorris MC, Paré M, and Everling S. On your mark, get set: brain stem circuitry underlying saccadic initiation. Can J Physiol Pharmacol 78: 934-944, 2000.

Munoz DP and Wurtz RH. Fixation cells in monkey superior colliculus. I. Characteristics of cell discharge. J Neurophysiol 70: 559-575, 1993.

Munoz DP and Wurtz RH. Saccade-related activity in monkey superior colliculus. I. Characteristics of burst and buildup cells. J Neurophysiol 73: 2313-2333, 1995.

Nambu A, Yoshida S, and Jinnai K. Movement-related activity of thalamic neurons with input from the globus pallidus and projection to the motor cortex in the monkey. Exp Brain Res 84: 279-284, 1991.

Olszewski J. The Thalamus of the Macaca mulatta: An Atlas for Use With the Stereotaxic Instrument. Basel, Switzerland: Karger, 1952, p. 54-70.

Paré M and Wurtz RH. Monkey posterior parietal cortex neurons antidromically activated from superior colliculus. J Neurophysiol 78: 3493-3497, 1997.

Paré M and Wurtz RH. Progression in neuronal processing for saccadic eye movements from parietal cortex area LIP to superior colliculus. J Neurophysiol 85: 2545-2562, 2001.

Ramcharan EJ, Cox CL, Zhan XJ, Sherman SM, and Gnadt JW. Cellular mechanisms underlying activity patterns in the monkey thalamus during visual behavior. J Neurophysiol 84: 1982-1987, 2000a.

Ramcharan EJ, Gnadt JW, and Sherman SM. Burst and tonic firing in thalamic cells of unanesthetized, behaving monkeys. Vis Neurosci 17: 55-62, 2000b.

Raybourn MS and Keller EL. Colliculo-reticular organization in primate oculomotor system. J Neurophysiol 40: 861-878, 1977.

Robinson DA. Eye movements evoked by collicular stimulation in the alert monkey. Vision Res 12: 1795-1808, 1972.

Rowe MH and Fischer Q. Dynamic properties of retino-geniculate synapses in the cat. Vis Neurosci 18: 219-231, 2001.

Schall JD. Neuronal activity related to visually guided saccades in the frontal eye fields of rhesus monkeys: comparison with supplementary eye fields. J Neurophysiol 66: 559-579, 1991.

Schall JD. Visuomotor areas of the frontal lobe. In: Cerebral Cortex. Extrastriate Cortex in Primates, edited by Rockland $\mathrm{K}$, Kaas JH, and Peters A. New York: Plenum, 1997, vol. 12, p. 527-638.

Schall JD and Thompson KG. Neural selection and control of visually guided eye movements. Annu Rev Neurosci 22: 241-259, 1999.

Schlag J and Schlag-Rey M. Visuomotor functions of central thalamus in the monkey. II. Unit activity related to visual events, targeting and fixation. J Neurophysiol 51: 1175-1195, 1984.

Schlag-Rey M and Schlag J. The central thalamus. In: Reviews of Oculomotor Research. The Neurobiology of Saccadic Eye Movements, edited by Wurtz RH and Goldberg ME. Amsterdam: Elsevier, 1989, vol. 3, p. 361390.

Schlag-Rey M and Schlag J. Visuomotor functions of central thalamus in monkey. I. Unit activity related to spontaneous eye movements. J Neurophysiol 51: 1149-1174, 1984. 
Shinoda Y, Kano M, and Futami T. Synaptic organization of the cerebellothalamo-cerebral pathway in the cat. I. Projection of individual cerebellar nuclei to single pyramidal tract neurons in areas 4 and 6. Neurosci Res 2: 133-156, 1985a.

Shinoda Y, Futami T, and Kano M. Synaptic organization of the cerebellothalamo-cerebral pathway in the cat. II. Input-output organization of single thalamocortical neurons in the ventrolateral thalamus. Neurosci Res 2: 157-180, 1985b.

Singer W, Tretter F, and Cynader M. Organization of cat striate cortex: a correlation of receptive-field properties with afferent and efferent connections. J Neurophysiol 38: 1080-1098, 1975.

Sommer MA. The role of the thalamus in motor control. Curr Opin Neurobiol 13: 663-670, 2003.

Sommer MA and Wurtz RH. Frontal eye field neurons orthodromically activated from the superior colliculus. J Neurophysiol 80: 3331-3335, 1998

Sommer MA and Wurtz RH. Activity in the pathway from superior colliculus to frontal eye field: mediodorsal thalamic relay neurons. Soc Neurosci Abstr 26:292, 2000a.

Sommer MA and Wurtz RH. Composition and topographic organization of signals sent from the frontal eye field to the superior colliculus. J Neurophysiol 83: 1979-2001, 2000b.

Sommer MA and Wurtz RH. Frontal eye field sends delay activity related to movement, memory, and vision to the superior colliculus. J Neurophysiol 85: 1673-1685, 2001.

Sommer MA and Wurtz RH. A pathway in primate brain for internal monitoring of movements. Science 296: 1480-1482, 2002.

Sommer MA and Wurtz RH. The dialogue between cerebral cortex and superior colliculus: implications for saccadic target selection and corollary discharge. In: The Visual Neurosciences, edited by Chalupa LM and Werner JS. Cambridge, MA: MIT Press, 2004a, vol. 2, p. 1466-1484.

Sommer MA and Wurtz RH. What the brain stem tells the frontal cortex. II. Role of the SC-MD-FEF pathway in corollary discharge. J Neurophysiol 91: 1403-1423, 2004.

Sparks DL and Hartwich-Young R. The deep layers of the superior colliculus. In: Reviews of Oculomotor Research. The Neurobiology of Saccadic Eye Movements, edited by Wurtz RH and Goldberg ME. Amsterdam: Elsevier, 1989, vol. 3, p. 213-255.

Sperry RW. Neural basis of the spontaneous optokinetic response produced by visual inversion. J Comp Physiol Psychol 43: 482-489, 1950.

Steriade M, Jones EG, and McCormick DA. Thalamus: Organisation and Function. Amsterdam: Elsevier, 1997, vol. 1, p. 31-174.

Stone J and Dreher B. Projection of X- and Y-cells of the cat's lateral geniculate nucleus to areas 17 and 18 of visual cortex. J Neurophysiol 36: 551-567, 1973.

Suzuki H and Azuma M. Topographic studies on visual neurons in the dorsolateral prefrontal cortex of the monkey. Exp Brain Res 53: 47-58, 1983.
Tanaka M. Contribution of signals downstream from adaptation to saccade programming. J Neurophysiol 90: 2080-2086, 2003.

Tanibuchi I and Goldman-Rakic PS. Dissociation of spatial-, object-, and sound-coding neurons in the mediodorsal nucleus of the primate thalamus. J Neurophysiol 89: 1067-1077, 2003.

Tehovnik EJ. Electrical stimulation of neural tissue to evoke behavioral responses. J Neurosci Methods 65: 1-17, 1996.

Tehovnik EJ, Sommer MA, Chou I-H, Slocum WM, and Schiller PH. Eye fields in the frontal lobes of primates. Brain Res Rev 32: 413-448, 2000.

Toyama K, Matsunami K, Ohno T, and Tokashiki S. An intracellular study of neuronal organization in the visual cortex. Exp Brain Res 21: 45-66, 1974.

Treue S. Neural correlates of attention in primate visual cortex. Trends Neurosci 24: 295-300, 2001.

Usrey WM, Reppas JB, and Reid RC. Paired-spike interactions and synaptic efficacy of retinal inputs to the thalamus. Nature 395: 384-387, 1998.

von Helmholtz H. Helmholtz's Treatise on Physiological Optics, edited by Southall JPC. Bristol: Thommes, 2000, vol. 3, p. 242-281.

Walker AE. The medial thalamic nucleus. A comparative anatomical, physiological and clinical study of the nucleus medialis dorsalis thalami. J Comp Neurol 73: 87-115, 1940 .

Watanabe Y, Takeda K, and Funahashi S. A comparison of working memory-related activity between primate prefrontal and thalamic neurons. Soc Neurosci Abstr 26: 976, 2000.

Weyand TG and Gafka AC. Activity of neurons in area 6 of the cat during fixation and eye movements. Vis Neurosci 15: 123-140, 1998a.

Weyand TG and Gafka AC. Corticostriatal and corticotectal neurons in area 6 of the cat during fixation and eye movements. Vis Neurosci 15: 141-151, 1998 b.

Wurtz RH, Basso MA, Paré M, and Sommer MA. The superior colliculus and the cognitive control of movement. In: The New Cognitive Neurosciences, (2nd ed.), edited by Gazzaniga MS. Cambridge, MA: The MIT Press, 2000, p. 573-587.

Wurtz RH and Mohler CW. Enhancement of visual responses in monkey striate cortex and frontal eye fields. J Neurophysiol 39: 766-772, 1976.

Wurtz RH and Sommer MA. Activity in the pathway from superior colliculus to frontal eye field: tectothalamic neurons. Soc Neurosci Abstr 26: 969, 2000.

Wurtz RH, Sommer MA, Paré M, and Ferraina S. Signal transformations from cerebral cortex to superior colliculus for the generation of saccades. Vision Res 41: 3399-3412, 2001.

Wyder MT, Massoglia DP, and Stanford TR. Quantitative assessment of the timing and tuning of visual-related, saccade-related, and delay period activity in primate central thalamus. J Neurophysiol 90: 2029-2052, 2003.

Zhu JJ and Lo FS. Control of recurrent inhibition of the lateral posteriorpulvinar complex by afferents from the deep layers of the superior colliculus of the rabbit. J Neurophysiol 80: 1122-1131, 1998. 